\title{
Extracurricular activities, screen media activity, and sleep may be modifiable factors influencing children's cognitive functioning: Evidence from the ABCD study
}

Namik Kirlic, $\mathrm{PhD}^{1}$, Janna M. Colaizzi, $\mathrm{PhD}^{1}$, Kelly T. Cosgrove, MA ${ }^{1}$, Zsofia P. Cohen ${ }^{1}$, HungWen Yeh, $\mathrm{PhD}^{1,2}$, Florence Breslin, $\mathrm{MS}^{1}$, Amanda Sheffield Morris, $\mathrm{PhD}^{1,3}$, Robin Aupperle, $\mathrm{PhD}^{1,4}$, and Martin P. Paulus, $\mathrm{MD}^{1}$

${ }^{1}$ Laureate Institute for Brain Research, Tulsa, OK, USA; ${ }^{2}$ Health Services \& Outcomes Research, Children's Mercy Hospital, Kansas City, MO; ${ }^{3}$ Department of Human Development and Family Sciences, Oklahoma State University, Tulsa, OK; ${ }^{4}$ School of Community Medicine, University of Tulsa, Tulsa, OK

Address correspondence to: Namik Kirlic, PhD; Laureate Institute for Brain Research; 6655 South Yale Ave, Tulsa, OK, 74136; nkirlic@laureateinstitute.org; Telephone: 918-502-5747; Fax: 918-502-5135

Funding Sources: The Adolescent Brain Cognitive Development (ABCD) Study is supported by the National Institutes of Health $(\mathrm{NIH})$ and additional federal partners under awards U01DA041022, U01DA041028, U01DA041048, U01DA041089, U01DA041106, U01DA041117, U01DA041120, U01DA041134, U01DA041148, U01DA041156, U01DA041174, U24DA041123, and U24DA041147. The study is also supported by the National Heart, Lung, and Blood Institute, the National Institute of Neurological Disorders and Stroke, the National Institute on Minority Health and Health Disparities, the NIH Office of Behavioral and Social Sciences Research, the NIH Office of Research on Women's Health, the Centers for Disease Control and Prevention-Division of Violence Prevention, the National Institute of Justice, the Centers for Disease Control and Prevention-Division of Adolescent and School Health, the National Science Foundation, and the National Endowment for the Arts.

Conflict of Interest: The authors have no conflicts of interest relevant to this article to disclose.

Acknowledgments: The National Institute on Drug Abuse was involved in the design and conduct of the study and data management. The funders otherwise had no role in the analysis and interpretation of the data; preparation, review, or approval of the manuscript; and decision to submit the manuscript for publication. The ABCD Consortium investigators designed and implemented the study and/or provided data but did not necessarily participate in analysis or writing of this report. This article reflects the views of the authors and may not reflect the opinions or views of the NIH or ABCD Consortium investigators.

Data used in the preparation of this article were obtained from the ABCD Study (https://abcdstudy.org), held in the NIMH Data Archive. This is a multisite, longitudinal study designed to recruit more than 10,000 children aged 9 to 10 years and follow up with them over 10 years into early adulthood. The ABCD data repository grows and changes over time. A listing of participating sites and a complete listing of the study investigators can be found at https://abcdstudy.org/wp-content/uploads/2019/04/Consortium_Members.pdf. The ABCD data used in this report came from the NIMH Data Archive (doi:10.15154/1412097). Partial data from this manuscript has been presented at the American College of Neuropsychopharmacology 2019 Annual Meeting. 


\begin{abstract}
This study used a machine learning framework in conjunction with a large battery of measures from 9,718 children from the Adolescent Brain Cognitive Development (ABCD) study to identify factors associated with fluid cognitive functioning (FCF). The identified algorithm explained $14.74 \%$ of variance in FCF. Among the most important factors were those that replicated previous research (socioeconomic and mental health factors), and novel, potentially modifiable factors, including extracurricular involvement and screen media activity. Pragmatic interventions targeting these behaviors may enhance cognitive performance and also protect against the negative impact of a range of factors on cognitive performance in at-risk children. The longitudinal data from $\mathrm{ABCD}$ will be able to assess causality by examining how changes in these factors affect subsequent performance.
\end{abstract}

Keywords: children, socioeconomic status, fluid cognitive functioning, ABCD, screen media activity, extracurricular activities, sleep 
The capacity to learn, solve problems, and adapt to novel situations (fluid cognitive functioning $[\mathrm{FCF}])$ is vital for children's success in achieving positive developmental outcomes. FCF positively relates to self-regulation, academic achievement, and psychological well-being during childhood, adolescence, and into young adulthood (Blair \& Razza, 2007; McClelland, Acock, Piccinin, Rhea, \& Stallings, 2013; McClelland \& Cameron, 2012). Although adverse environments can have a sustained negative impact on FCF and its associated outcomes, the plasticity of the developing brain presents an opportunity to intervene and assert meaningful improvements in these domains. Therefore, research identifying factors contributing to FCF in childhood is essential for developing meaningful interventions to promote positive long-term outcomes.

FCF consists of broad abilities in executive function, attention, inhibition, processing speed, and memory, and represents a global assessment of one's ability to think, act, and learn. It is understood that FCF reaches its peak in early adulthood and declines across the remainder of the lifespan. However, a large number of factors influences performance on FCF measures, some of which are difficult to modify (e.g., parental education), while others lend themselves well to personal and/or systemic interventions (e.g., exercise).

Among the most robust contributors of poor FCF function in children are those associated with low socioeconomic status (SES) (Farah et al., 2006; Noble, Norman, \& Farah, 2005). These effects are lasting, as individuals who experience childhood poverty exhibit working memory deficits as adults (Evans and Schamberg, 2009). On the other hand, higher FCF can act as a protective factor in socioeconomic disparity as it is often a mediator between SES and individual outcomes (Lawson et al, 2018). Stress exposure often co-occurs with low SES, and in fact may mediate the relation between poverty and executive function in children 
(Piccolo, Sbicigo, Grassi-Oliveira, \& Fumagalli de Salles, 2014). Research shows that negative parenting practices and household chaos lead to increased levels of stress during childhood and result in FCF deficits, even when controlling for the effects of poverty (Vernon-Feagans, Willoughby, \& Garrett-Peters, 2016). Furthermore, early life stress (e.g., childhood maltreatment, parental psychopathology, and family dysfunction) in particular can have detrimental effects on cognitive and executive function across the lifespan (Irigaray et al., 2013). Factors related to mental and physical health are also frequently implicated in studies of childhood FCF. FCF deficits are commonly seen in neurodevelopmental [e.g., ADHD; (Biederman et al., 2004)] and mood and anxiety disorders (Goodall et al., 2018; Kavanaugh \& Holler, 2014). Furthermore, children who receive insufficient sleep (i.e., fewer than the recommended number of hours for their age) are more likely to receive poorer scores on motherand teacher-reported measures of FCF (Taveras, Rifas-Shiman, Bub, Gillman, \& Oken, 2017). Finally, obese children and adolescents exhibit significantly poorer inhibitory control than those with healthy weight (Reinert, Po'e, \& Barkin, 2013), suggesting that there is a relation between physical health and FCF. Taken together, these data show the multitude of environmental and behavioral factors that demonstrate a relation with FCF during development. Further, they emphasize the need to identify factors that may impact FCF in order to guide potential targeted interventions.

Despite significant research efforts, our knowledge of factors associated with FCF remains limited. This is in part due to studies with small sample sizes and over-reliance on traditional statistical approaches limited in their ability to examine large sets of unique variables simultaneously. This has impeded the development of interventions that may meaningfully impact FCF deficits in children. Given that pathophysiology of FCF deficits is complex and 
likely influenced by numerous factors of potentially shared variance, sophisticated multivariate statistical models employing large datasets are necessary to identify generalizable associations with FCF and factors of adequate prospective clinical utility. Machine learning (ML) approaches address these issues across a number of different fields as they allow for the simultaneous testing of numerous factors and their complex interactions, and for non-linearity in producing predictive models (McArdle \& Ritschard, 2013). Although ML approaches with cross-sectional data cannot infer causation, by iteratively testing all possible relations and identifying a set of important associations, they can improve upon theory-driven definitions of associations between important factors and FCF.

With a large, diverse dataset of children and variables across a number of broad domains relevant to cognitive function, the Adolescent Brain Cognitive Development (ABCD) study is uniquely positioned to comprehensively assess factors associated with FCF. Therefore, this study used a ML framework to examine the associations between a range of variables associated with baseline FCF across demographic/environmental, mental and physical health, and behavioral domains. The aims were to identify whether algorithms developed based on these measures may identify important factors associated with FCF, and to identify factors that could potentially be targeted with scalable prevention and intervention efforts and explored in longitudinal analyses.

\section{METHODS}

\section{Participants}

The ABCD study is large multi-site, longitudinal study following 9-10-year-old children over a 10-year period. It is designed to assess developmental changes across a comprehensive range of domains, including brain structure and function, cognition, behavior, and mental health 
(Jernigan, Brown, \& Dowling, 2018). The current report examines the complete baseline sample $(\mathrm{N}=11,877$; Data release 2.0.1). Informed consent and assent were obtained from a parent or legal guardian and the child, respectively, and all procedures were approved by a central Institutional Review Board. The University of California, San Diego, institutional review board has indicated that analyses using the publicly released ABCD Study data are not human subjects research and therefore do not require their own approval.

We excluded from the analysis participants without data for the FCF composite score. Next, in order to limit dependence in the response variable, we randomly selected one child per family where multiple siblings were assessed, resulting in a total of 9,718 participants.

\section{Measures}

Fluid Cognitive Functioning. FCF was measured using the NIH Toolbox Neurocognitive Battery specified for ages 7-17 (Luciana et al., 2018). The age-corrected composite FCF score consists of the following tasks: Dimensional Change Card Sort measuring ability to plan, organize, and execute goal-directed behaviors; Flanker Inhibitory Control and Attention measuring the ability to handle multiple environmental stimuli; Picture Sequence Memory measuring episodic memory including the acquisition, storage, and retrieval of information; List Sorting Working Memory measuring the ability to store, manipulate, and hold new information; and Pattern Comparison Processing Speed measuring the processing ability of new information within a certain amount of time.

Demographic and Environmental Information. Parents reported demographic information regarding household and immediate family members using the PhenX survey toolkit (Barch et al., 2018). SES was measured using the relative income disparity, or the area deprivation index 
(Singh, 2003), and a subscale for basic needs unaffordability (Diemer, Mistry, Wadsworth, Lopez, \& Reimers, 2012). Neighborhood crime rates based on US Census data (Bagot et al., 2018), PhenX Toolkit subscales for the perceived safety and presence of crime in one's neighborhood (Safety from Crime subscale) (Zucker et al., 2018), and child accessibility to substances (Community Risk and Protective Factors subscale) (Lisdahl et al., 2018) were provided. Child trauma exposure was measured from a subscale of the Kiddie Schedule for Affective Disorders and Schizophrenia for DSM-5 (Barch et al., 2018).

Mental Health and Familial Variables. The Achenbach Adult Self-Report Questionnaire (Barch et al., 2018) provided parental psychopathology and adaptive functioning scores. The Family History Assessment Module Screener (Barch et al., 2018) reported history of psychopathology among first- and second-degree relatives, and the Family Environment Scale (Zucker et al., 2018) assessed family conflict. Family conflict was also assessed via child report with the FES. Child perception of parental monitoring and parental acceptance were measured with the Parental Monitoring Scale (Zucker et al., 2018) and the Acceptance Subscale of the Child Report of Behavior Inventory (Zucker et al., 2018).

Child Mental Health Symptoms and Behaviors. Parents reported child dimensional psychopathology using the Achenbach Child Behavior Checklist (Barch et al., 2018), while Parent General Behavior Inventory for Children and Adolescents (Barch et al., 2018) assessed mania symptoms. Sleep Disturbances Scale for Children (Bruni et al., 1996) provided sleep behaviors, and extracurricular activities and physical exercise was assessed with the Sports and Activities Involvement Questionnaire (Huppertz et al., 2016).

Child self-reports included the four subscales of the Behavioral Inhibition \& Behavioral Activation Scales (Barch et al., 2018) and the Urgency, Premeditation, Perseverance, Sensation 
Seeking, Positive Urgency subscales on the Impulsive Behavior Scale for Children (Barch et al., 2018). Estimated amounts of time typically spent engaging in various screen media activity (Bagot et al., 2018) were reported. Physical exercise was assessed using the Youth Risk Behavior Survey (CDC, 2016). Objective physical health measures of body mass index and waist circumference were collected using the National Health and Nutrition Examination Survey (CDC (Division of Nutrition, 2016). For additional information, see the supplemental information.

\section{Analysis}

Statistical analyses were conducted in R 3.5.1 using RStudio (Team, 2015). Descriptive statistics were obtained using the R package 'psych' (Revelle, 2017).

Machine learning. In order to build a model that would best characterize the agecorrected FCF composite scores, 52 unique variables derived from the above measures were submitted to ML analyses.

ML algorithms rely on unique assumptions and may result in variations in prediction accuracy, but no single algorithm is known to always outperform others on predictive accuracy. We therefore chose to use the "wisdom of crowds" approach (Marbach et al., 2012), which combines predictions from multiple base learners (prediction algorithms). We utilized multiple "out of box" ML methods, followed by combining the predictions across methods by stacking or meta ensemble (Breiman, 1996; Van der Laan, Polley, \& Hubbard, 2007; Wolpert, 1992). A nested cross-validation (nCV), in which the inner loop is used to build base and stacked models and the outer loop to evaluate model performance, was conducted to assess performance of stack ensembles in independent, unseen datasets. The nCV procedure was repeated five times to quantify the variability of prediction accuracy and variable importance. 
We applied four base learners in the inner loop for each training set, including elastic net(Barsaglini, Sartori, Benetti, Pettersson-Yeo, \& Mechelli), support vector regression (SVR) (Suykens \& Vandewalle, 1999), conditional random forest (CRF) (Hothorn, Hornik, \& Zeileis, 2006), and k-Nearest Neighbors (knn) (Keller, Gray, \& Givens, 1985). For each base learner, the tuning parameter(s) were optimized by 5 -fold cross validation. Specifically, each training set was partitioned into five distinct subsets, where four subsets were used for the training process to evaluate different hyper parameter values; as a pre-process procedure, predictors with lower than $10 \%$ variability were removed and the remaining variables were standardized to 0 mean and unit variance. Optimal hyper-parameter values were chosen through random search (Bergstra \& Bengio, 2012) and the "best" rule (Hastie, Tibshirani, \& Friedman, 2009) using $R^{2}$ as the model performance metric. Next, we obtained four sets of predicted values, one from each base leaner and their corresponding optimal hyper-parameter values.

Within the inner cross validation loop, each method produced a single best model and $R^{2}$ of the training sample (training $R^{2}$ ). A stacked model was built by taking the arithmetic mean of predictions from each base learner, weighted by each model's training $R^{2}$. In the outer loop, we applied the stacked model to predict the response in the corresponding validation set. Predicted values of the validation sets were combined and compared with the observed values to compute $R^{2}$. With five replications of partitions, we summarized the performance by the mean and $95 \%$ confidence interval of $R^{2}$.

We assessed variable importance (VI) using stacking, where each base model provided importance for each feature. The different individual methods had varying VI measures: absolute values of regression coefficients for elastic net, an "out-of-bag" mean square error obtained by permutation for CRF, and a "filter" approach for SVR and knn wherein the response variable 
was regressed on each feature one at a time by a loess (Locally Weighted Scatter-plot Smoother), and the $R^{2}$ was computed as the variable importance. VI measures were scaled between 0 and 100 for each individual model. The stacked importance was computed as the weighted average of the importance across models using the weights determined by the stacking model described above. This produced a single set of VI values for each stacked model in the outer loop of nCV. VI was averaged across folds to obtain a single set of values. Five random partitions were used (five repeats of $\mathrm{nCV}$ ), and 95\% confidence intervals for VI taken as each variable's mean importance \pm 1.96 times its standard deviation. We computed Spearman correlation coefficients and FDR corrected p-values for comparison purpose (Table S2). Analyses for prediction models were implemented using the 'caret' package (Kuhn, 2008) and marginal relations between FCF and each predictor was assessed by partial-dependence plots ('pdp' package) (Greenwell, 2017). For additional information, see the Supplementary methods.

Follow-up analyses. We conducted follow-up analyses using linear mixed effects [LME; R-package 'lme4' (Bates, Maechler, Bolker, \& Walker, 2014)] models on variables constituting potentially modifiable behaviors, including weekday screen media activity and extracurricular involvement, to further delineate their effects on FCF. Regarding weekday screen media, type of activities were entered as fixed effects with $\mathrm{ABCD}$ site entered as random effect. Regarding extracurricular involvement, the numerous activities were combined into three distinct categories: individual physical, group physical, and art-related activities and entered as fixed effects with $A B C D$ site entered as a random effect. Given the large sample size, the analysis focused on effect sizes rather than statistically significant relations.

\section{RESULTS}


Table 1 presents demographic and clinical characteristics of the final sample. On average, participants evidenced FCF in the normal range (mean=95.57, sd=17.48; Figure S3).

\section{Machine Learning}

ML analysis identified a model with 47 variables that explained $14.74 \%$ of variance $(95 \%$ CI: 14.53-14.88\%) in FCF (Figure S4, Table 2). Of the top 15 variables with the highest importance (Figure 1), those negatively related to FCF were (1) socioeconomic: family income less than $\$ 50,000$, relative income disparity, racial minority status, and unaffordability of basic needs; and (2) child-specific: motivation to follow one's goals, symptoms of mania, weekday screen media activity, positive urgency, externalizing behaviors, and lack of perseverance. Variables positively related to FCF were (1) demographic/environmental: parents married, parents living together, and increased neighborhood safety; and (2) child-specific: extracurricular involvement and sleep duration. Figure 2 shows the partial dependence (PD) plots of marginal effect each variable with the highest importance has on FCF. PD plots indicated a non-linear relation between FCF and extracurricular involvement, weekday screen time, neighborhood safety and crime, and basic needs unaffordability.

\section{Follow-up analyses}

LME analysis revealed a small positive relation between FCF and art-related $(\mathrm{F}(1,9490)=75.90, \mathrm{p}<.001 ;$ Cohen's $\mathrm{d}=.18)$ and team $(\mathrm{F}(1,9494)=42.32, \mathrm{p}<.001$; Cohen's $\mathrm{d}=.13)$ and individual sport $(\mathrm{F}(1,9492)=30.24, \mathrm{p}<.001$; Cohen's d=.11) activities (Figure S5).

LME analysis revealed a small negative relation between FCF and weekday time spent watching internet videos $(\mathrm{F}(1,9673)=59.95, \mathrm{p}<.001$; Cohen's $\mathrm{d}=.16)$, playing mature-rated video 
games $(\mathrm{F}(1,9673)=33.51, \mathrm{p}<.001$; Cohen's $\mathrm{d}=.12)$, watching TV shows and movies

$(F(1,9672)=29.28, p<.001$; Cohen's d $=.11)$, video chatting $(F(1,9670)=27.18, p<.001$; Cohen's $\mathrm{d}=.11)$, watching mature-rated movies $\operatorname{FCF}(\mathrm{F}(1,9670)=12.69, \mathrm{p}<.001$; Cohen's $\mathrm{d}=.07)$, and using social media $(F(1,9664)=6.79, p<.01 ;$ Cohen's $d=.05)$, whereas time spent playing video games $(\mathrm{F}(1,9669)=0.13, \mathrm{p}=.72$; Cohen's $d=.01)$ and texting $(\mathrm{F}(1,9669)=0.10, \mathrm{p}=.75$; Cohen's $\mathrm{d}=.01$ ) did not show a relation with FCF (Figure S5).

\section{DISCUSSION}

To our knowledge, this study is the first to use a ML framework to examine associations between a large number of unique demographic, environmental, and child factors and FCF in a large prepubertal group of youth. This is an important task given that FCF is associated with long-term psychological, academic, and occupational outcomes. We observed a normally distributed range of FCF scores in this large sample of 9,718 children from across the U.S. Notably, a significant percentage (6.3\%) performed in the extremely low range, while an additional $11.8 \%$ performed in the borderline range.

Fifteen variables with highest importance associated with FCF that were identified by the ML analysis fell into two broad categories: socioeconomic and child-specific. First, the findings confirm previous reports (Lawson, Hook, \& Farah, 2018), and further underscore the significant adverse effect of socioeconomic deprivation on FCF. Our findings extend previous literature beyond measures of income and education, further highlighting the adverse impact of relative income disparity and unaffordability of basic needs, such as food, residence, and medical care. The associated chronic stress may mediate the relation between poverty and FCF (Blair et al., 2011; Piccolo et al., 2014). For example, research shows that negative parenting and household 
chaos lead to heightened stress exposure during childhood and result in FCF deficits, even when controlling for the direct effects of poverty (Vernon-Feagans et al., 2016). Childhood maltreatment, parental psychopathology, and family dysfunction in particular can have detrimental effects on cognitive and executive function across the lifespan (Irigaray et al., 2013). Therefore, impoverished circumstances may create an environment characterized by chronic stress, which, in turn, has a lasting impact on the neurobiological foundations of cognitive development (Evans \& Schamberg, 2009; Lawson et al., 2018).

Psychopathology closely follows exposure to chronic stress (Kessler et al., 2010). Our findings support previous work indicating that impairment in processes underlying irritability, changes in mood, and aggressive and rule-breaking behavior, such as effortful control, selfregulation, and impulse control relate to impairments in FCF (Gartstein \& Fagot, 2003; Horn, Roessner, \& Holtmann, 2011; Mezzacappa, Kindlon, \& Earls, 1999). Poor sleep habits in children, often a consequence of poverty and environmental stress (Williamson \& Mindell, 2019), may also add to a set of existing risk factors for poor FCF. For example, child with insufficient sleep evidence deficits in inhibitory responses, working memory, executive function, and emotional control (Taveras et al., 2017). Together, co-occurring socioeconomic disadvantages, adverse environments, and mental health problems may have a significant adverse effect on the neurobiological development and functioning in developing children.

Conversely, there is converging evidence that involvement in constructive, non-academic activity accompanies persistent positive and potentially dose-response outcomes in cognitive performance and academic achievement (Eccles, Barber, Stone, \& Hunt, 2003; Fredricks, 2012). Our data, however, point to a non-linear relation between these variables and suggest that there may be a point of diminishing returns. Nevertheless, participation in arts is associated with 
improvement in a range of cognitive domains (Rauscher, Shaw, \& Ky, 1993; Schellenberg, 2004; Winsler, Gara, Alegrado, Castro, \& Tavassolie, 2019), while participation in physical activities and organized, supervised sports has been associated with increased self-regulation, inhibition, and problem solving (Jacobson \& Matthaeus, 2014; Piché, Fitzpatrick, \& Pagani, 2015). Extracurricular enrichment may promote new learning and skill acquisition, creativity, self-efficacy, formation of strong social bonds with non-familial adults and peers, identity development, and physical health (Eccles et al., 2003; Etnier \& Chang, 2009), which may translate into improved cognitive performances and educational attainment (Jacobson \& Matthaeus, 2014), even in at-risk children (Peck, Roeser, Zarrett, \& Eccles, 2008).

The research on the impact of screen media activity on development is complex. A recent report showed that increased screen media activity generally has a negative impact on sleep quality and duration, which in turn affects problem behaviors (Guerrero, Barnes, Chaput, \& Tremblay, 2019), and both are known to be negatively associated with FCF (Short \& Chee, 2019; Taveras et al., 2017). A recent study with the first half of the ABCD sample examining the direct relation between FCF and screen media activity found that gaming activities had a positive relation with FCF, while social media activities related negatively to FCF (Paulus et al., 2019). Our data on the full sample also suggest that different types of screen media activity may have divergent effects on cognitive function, specifically that time spent watching TV, movies, and internet videos and playing mature-rated video games had the strongest dose-response negative relation with FCF.

Although contributors of FCF are multifaceted, extracurricular enrichment, screen media activity, and sleep may be targeted by pragmatic, scalable, and systemic interventions. Psychoeducation with parents and children on these and related topics may result in significant 
behavioral changes. Indeed, one study found that psychoeducation targeting sleep habits had not only significant sustained effects on sleep behaviors, but was also associated with subsequent improvements in cognitive performance (Rey, Guignard-Perret, Imler-Weber, Garcia-Larrea, \& Mazza, 2020). Further, schools could increase participation in extracurricular activities among children by making them widely available and accessible. Such interventions resulting even in marginal improvements in FCF may translate into increased long-term scholastic performance and psychosocial outcomes. Importantly, it may be the case that targeting these factors increases resilience and cognitive performance among children particularly at risk for deficits in FCF secondary to exposure to adverse environments.

\section{Limitations}

The results presented here are cross-sectional. Therefore, causality cannot be inferred, and bi-directional relations between FCF and associated factors are possible. Although we employed repeated $\mathrm{nCV}$ to reduce the risk of over-estimation of prediction accuracy, an external validation of the model with an independent sample would be useful in demonstrating its replicability. We only included data from the baseline assessment of these children. Additional research will be able to assess the predictive value of these factors in later years and at later developmental stages.

\section{Conclusions}

We used a machine learning framework to identify variables associated with cognitive functioning in a large sample of children. In addition to socioeconomic disparity factors previously associated with poor cognitive functioning, we identified a number of novel and 
potentially modifiable behavioral factors, including participation in extracurricular activities, screen media time activity, and sleep duration. Modifying these behaviors may not only serve to enhance cognitive performance but may also constitute protective factors against the negative impact of socioeconomic, environmental, and mental health factors on cognitive performance in at-risk children. The longitudinal data from $\mathrm{ABCD}$ will be able to begin to assess causality by examining how changes in these factors affect subsequent cognitive performance. 


\section{REFERENCES}

Bagot, K. S., Matthews, S. A., Mason, M., Squeglia, L. M., Fowler, J., Gray, K., .. Patrick, K. (2018). Current, future and potential use of mobile and wearable technologies and social media data in the ABCD study to increase understanding of contributors to child health. Dev Cogn Neurosci, 32, 121-129. Retrieved from https://www.ncbi.nlm.nih.gov/pubmed/29636283. doi:10.1016/j.den.2018.03.008

Barch, D. M., Albaugh, M. D., Avenevoli, S., Chang, L., Clark, D. B., Glantz, M. D., .. . Sher, K. J. (2018). Demographic, physical and mental health assessments in the adolescent brain and cognitive development study: Rationale and description. Dev Cogn Neurosci, 32, 55-66. Retrieved from https://www.ncbi.nlm.nih.gov/pubmed/29113758. doi:10.1016/j.den.2017.10.010

Barsaglini, A., Sartori, G., Benetti, S., Pettersson-Yeo, W., \& Mechelli, A. (2014). The effects of psychotherapy on brain function: a systematic and critical review. Progress in Neurobiology., 114, 1-14.

Bates, D., Maechler, M., Bolker, B., \& Walker, S. (2014). 1me4: Linear mixed-effects models using S4 classes. R package version 1.1-7. URL http://CRAN. $R$-project. org/package= lme4.

Bergstra, J., \& Bengio, Y. (2012). Random search for hyper-parameter optimization. Journal of Machine Learning Research, 13(Feb), 281-305.

Biederman, J., Monuteaux, M. C., Doyle, A. E., Seidman, L. J., Wilens, T. E., Ferrero, F., .. . Faraone, S. V. (2004). Impact of executive function deficits and attentiondeficit/hyperactivity disorder (ADHD) on academic outcomes in children. Journal of consulting and clinical psychology, 72(5), 757. 
Blair, C., Granger, D. A., Willoughby, M., Mills-Koonce, R., Cox, M., Greenberg, M. T., .. . Investigators, F. (2011). Salivary cortisol mediates effects of poverty and parenting on executive functions in early childhood. Child Development, 82(6), 1970-1984.

Blair, C., \& Razza, R. P. (2007). Relating effortful control, executive function, and false belief understanding to emerging math and literacy ability in kindergarten. Child development, $78(2), 647-663$.

Breiman, L. (1996). Stacked regressions. Machine learning, 24(1), 49-64.

Bruni, O., Ottaviano, S., Guidetti, V., Romoli, M., Innocenzi, M., Cortesi, F., \& Giannotti, F. (1996). The Sleep Disturbance Scale for Children (SDSC). Construction and validation of an instrument to evaluate sleep disturbances in childhood and adolescence. J. Sleep Res, 5(4), 251-261.

CDC. (2016). Youth Risk Behavior Survey. Retrieved from https://www.cdc.gov/healthyyouth/data/yrbs/

CDC (Division of Nutrition, P. A. a. O. (2016). Anthropometry Procedures Manual. Retrieved from https://wwwn.cdc.gov/nchs/data/nhanes/2015-2016/manuals/2016 Anthropometry_Procedures_Manual.pdf.

Diemer, M. A., Mistry, R. S., Wadsworth, M. E., Lopez, I., \& Reimers, F. (2012). Best practices in conceptualizing and measure social class in psycholgocial research. Anal. Soc. Issues Public Policy, 13(1), 77-113.

Eccles, J. S., Barber, B. L., Stone, M., \& Hunt, J. (2003). Extracurricular activities and adolescent development. Journal of Social Issues, 59(4), 865-889. 
Etnier, J. L., \& Chang, Y.-K. (2009). The effect of physical activity on executive function: a brief commentary on definitions, measurement issues, and the current state of the literature. Journal of Sport and Exercise Psychology, 31(4), 469-483.

Evans, G. W., \& Schamberg, M. A. (2009). Childhood poverty, chronic stress, and adult working memory. Proc Natl Acad Sci U S A, 106(16), 6545-6549. Retrieved from https://www.ncbi.nlm.nih.gov/pubmed/19332779. doi:10.1073/pnas.0811910106

Farah, M. J., Shera, D. M., Savage, J. H., Betancourt, L., Giannetta, J. M., Brodsky, N. L., . . . Hurt, H. (2006). Childhood poverty: Specific associations with neurocognitive development. Brain research, 1110(1), 166-174.

Fredricks, J. A. (2012). Extracurricular participation and academic outcomes: Testing the overscheduling hypothesis. Journal of Youth and Adolescence, 41(3), 295-306.

Gartstein, M. A., \& Fagot, B. I. (2003). Parental depression, parenting and family adjustment, and child effortful control: Explaining externalizing behaviors for preschool children. Journal of Applied Developmental Psychology, 24(2), 143-177.

Goodall, J., Fisher, C., Hetrick, S., Phillips, L., Parrish, E. M., \& Allott, K. (2018).

Neurocognitive functioning in depressed young people: A systematic review and metaanalysis. Neuropsychology review, 28(2), 216-231.

Greenwell, B. M. (2017). pdp: an R Package for constructing partial dependence plots. The $R$ Journal, 9(1), 421-436.

Guerrero, M. D., Barnes, J. D., Chaput, J.-P., \& Tremblay, M. S. (2019). Screen time and problem behaviors in children: exploring the mediating role of sleep duration. International Journal of Behavioral Nutrition and Physical Activity, 16(1), 105. 
Hastie, T., Tibshirani, R., \& Friedman, J. (2009). The elements of statistical learning 2nd edition. In: New York: Springer.

Horn, K., Roessner, V., \& Holtmann, M. (2011). Neurocognitive performance in children and adolescents with bipolar disorder: a review. European child \& adolescent psychiatry, 20(9), 433.

Hothorn, T., Hornik, K., \& Zeileis, A. (2006). Unbiased recursive partitioning: A conditional inference framework. Journal of Computational and Graphical statistics, 15(3), 651-674.

Huppertz, C., Bartels, M., de Zeeuw, E. L., van Beijsterveldt, C. E. M., Hudziak, J. J., Willemsen, G., . . . de Geus, E. J. C. (2016). Individual differences in exercise behavior: stability and change in genetic and environmental determinants from age 7-18. Behav. Genet., 46(5), 665-679.

Irigaray, T. Q., Pacheco, J. B., Grassi-Oliveira, R., Fonseca, R. P., Leite, J. C. d. C., \& Kristensen, C. H. (2013). Child maltreatment and later cognitive functioning: a systematic review. Psicologia: Reflexão e Crítica, 26(2), 376-387.

Jacobson, J., \& Matthaeus, L. (2014). Athletics and executive functioning: How athletic participation and sport type correlate with cognitive performance. Psychology of Sport and Exercise, 15(5), 521-527.

Jernigan, T. L., Brown, S. A., \& Dowling, G. J. (2018). The Adolescent Brain Cognitive Development Study. Journal of research on adolescence: the official journal of the Society for Research on Adolescence, 28(1), 154-156.

Kavanaugh, B., \& Holler, K. (2014). Executive, emotional, and language functioning following childhood maltreatment and the influence of pediatric PTSD. Journal of Child \& Adolescent Trauma, 7(2), 121-130. 
Keller, J. M., Gray, M. R., \& Givens, J. A. (1985). A fuzzy k-nearest neighbor algorithm. IEEE transactions on systems, man, and cybernetics(4), 580-585.

Kessler, R. C., McLaughlin, K. A., Green, J. G., Gruber, M. J., Sampson, N. A., Zaslavsky, A. M., ... Angermeyer, M. (2010). Childhood adversities and adult psychopathology in the WHO World Mental Health Surveys. The British Journal of Psychiatry, 197(5), 378-385.

Kuhn, M. (2008). Caret package. Journal of Statistical Software, 28(5), 1-26.

Lawson, G. M., Hook, C. J., \& Farah, M. J. (2018). A meta-analysis of the relationship between socioeconomic status and executive function performance among children. Dev Sci, 21(2). Retrieved from https://www.ncbi.nlm.nih.gov/pubmed/28557154. doi:10.1111/desc. 12529

Lisdahl, K. M., Sher, K. J., Conway, K. P., Gonzalez, R., Feldstein Ewing, S. W., Nixon, S. J., . . . Heitzeg, M. (2018). Adolescent brain cognitive development (ABCD) study: Overview of substance use assessment methods. Dev Cogn Neurosci, 32, 80-96. Retrieved from https://www.ncbi.nlm.nih.gov/pubmed/29559216. doi:10.1016/j.den.2018.02.007

Luciana, M., Bjork, J. M., Nagel, B. J., Barch, D. M., Gonzalez, R., Nixon, S. J., \& Banich, M. T. (2018). Adolescent neurocognitive development and impacts of substance use: Overview of the adolescent brain cognitive development (ABCD) baseline neurocognition battery. Dev Cogn Neurosci, 32, 67-79. Retrieved from https://www.ncbi.nlm.nih.gov/pubmed/29525452. doi:10.1016/j.dcn.2018.02.006

Marbach, D., Costello, J. C., Küffner, R., Vega, N. M., Prill, R. J., Camacho, D. M., . . Chen, Y. (2012). Wisdom of crowds for robust gene network inference. Nature methods, 9(8), 796. McArdle, J. J., \& Ritschard, G. (2013). Contemporary issues in exploratory data mining in the behavioral sciences: Routledge. 
McClelland, M. M., Acock, A. C., Piccinin, A., Rhea, S. A., \& Stallings, M. C. (2013). Relations between preschool attention span-persistence and age 25 educational outcomes. Early childhood research quarterly, 28(2), 314-324.

McClelland, M. M., \& Cameron, C. E. (2012). Self-regulation in early childhood: Improving conceptual clarity and developing ecologically valid measures. Child development perspectives, 6(2), 136-142.

Mezzacappa, E., Kindlon, D., \& Earls, F. (1999). Relations of age to cognitive and motivational elements of impulse control in boys with and without externalizing behavior problems. Journal of abnormal child psychology, 27(6), 473-483.

Noble, K. G., Norman, M. F., \& Farah, M. J. (2005). Neurocognitive correlates of socioeconomic status in kindergarten children. Developmental science, 8(1), 74-87.

Paulus, M. P., Squeglia, L. M., Bagot, K., Jacobus, J., Kuplicki, R., Breslin, F. J., . . Bartsch, H. (2019). Screen media activity and brain structure in youth: Evidence for diverse structural correlation networks from the ABCD study. Neuroimage, 185, 140-153.

Peck, S. C., Roeser, R. W., Zarrett, N., \& Eccles, J. S. (2008). Exploring the roles of extracurricular activity quantity and quality in the educational resilience of vulnerable adolescents: Variable-and pattern-centered approaches. Journal of Social Issues, 64(1), 135-156.

Piccolo, L. d. R., Sbicigo, J. B., Grassi-Oliveira, R., \& Fumagalli de Salles, J. (2014). Do socioeconomic status and stress reactivity really impact neurocognitive performance? Psychology \& Neuroscience, 7(4), 567. 
Piché, G., Fitzpatrick, C., \& Pagani, L. S. (2015). Associations between extracurricular activity and self-regulation: a longitudinal study from 5 to 10 years of age. American Journal of Health Promotion, 30(1), e32-e40.

Rauscher, F. H., Shaw, G. L., \& Ky, C. N. (1993). Music and spatial task performance. Nature, 365(6447), 611-611.

Reinert, K. R. S., Po'e, E. K., \& Barkin, S. L. (2013). The relationship between executive function and obesity in children and adolescents: a systematic literature review. Journal of obesity, 2013, 820956-820956. Retrieved from https://pubmed.ncbi.nlm.nih.gov/23533726

https://www.ncbi.nlm.nih.gov/pmc/articles/PMC3595670/. doi:10.1155/2013/820956

Revelle, W. R. (2017). psych: Procedures for personality and psychological research.

Rey, A. E., Guignard-Perret, A., Imler-Weber, F., Garcia-Larrea, L., \& Mazza, S. (2020). Improving sleep, cognitive functioning and academic performance with sleep education at school in children. Learning and Instruction, 65, 101270.

Schellenberg, E. G. (2004). Music lessons enhance IQ. Psychological Science, 15(8), 511-514.

Short, M. A., \& Chee, M. W. (2019). Adolescent sleep restriction effects on cognition and mood. Progress in Brain Research, 246, 55-71.

Singh, G. K. (2003). Area deprivation and widening inequalities in US mortality, 1969-1998. Am J Public Health, 93, 1137-1143.

Suykens, J. A., \& Vandewalle, J. (1999). Least squares support vector machine classifiers. Neural processing letters, 9(3), 293-300. 
Taveras, E. M., Rifas-Shiman, S. L., Bub, K. L., Gillman, M. W., \& Oken, E. (2017).

Prospective study of insufficient sleep and neurobehavioral functioning among schoolage children. Academic pediatrics, 17(6), 625-632.

Team, R. (2015). RStudio: integrated development for R. RStudio. Inc., Boston, MA, 639, 640.

Van der Laan, M. J., Polley, E. C., \& Hubbard, A. E. (2007). Super learner. Statistical applications in genetics and molecular biology, 6(1).

Vernon-Feagans, L., Willoughby, M., \& Garrett-Peters, P. (2016). Predictors of behavioral regulation in kindergarten: household chaos, parenting, and early executive functions. Developmental psychology, 52(3), 430.

Williamson, A. A., \& Mindell, J. A. (2019). Cumulative socio-demographic risk factors and sleep outcomes in early childhood. sleep.

Winsler, A., Gara, T. V., Alegrado, A., Castro, S., \& Tavassolie, T. (2019). Selection into, and academic benefits from, arts-related courses in middle school among low-income, ethnically diverse youth. Psychology of Aesthetics, Creativity, and the Arts.

Wolpert, D. H. (1992). Stacked generalization. Neural networks, 5(2), 241-259.

Zucker, R. A., Gonzalez, R., Feldstein Ewing, S. W., Paulus, M. P., Arroyo, J., Fuligni, A., .. . Wills, T. (2018). Assessment of culture and environment in the Adolescent Brain and Cognitive Development Study: Rationale, description of measures, and early data. Dev Cogn Neurosci, 32, 107-120. Retrieved from https://www.ncbi.nlm.nih.gov/pubmed/29627333. doi:10.1016/j.den.2018.03.004 


\section{TABLES}

Table 1. Sample characteristics.

\begin{tabular}{|c|c|c|}
\hline Demographics (parent report) & $\mathrm{n}=9718$ & Range \\
\hline Age, Mean (SD) & $9.89(0.62)$ & $9.00-10.92$ \\
\hline Gender, N (\%) & & \\
\hline Male & $5125(52.8)$ & \\
\hline Female & $4590(47.2)$ & \\
\hline Race, N (\%) & & \\
\hline White & $4937(50.9)$ & \\
\hline Black or African American & $1459(15.0)$ & \\
\hline Hispanic & $2066(21.3)$ & \\
\hline Asian & $225(2.3)$ & \\
\hline Other/Mixed & $1019(10.5)$ & \\
\hline Twin Status, N (\%) & & \\
\hline Yes & $856(8.8)$ & \\
\hline No & $8862(91.2)$ & \\
\hline Siblings in Household, N (\%) & & \\
\hline Yes & $1835(18.9)$ & \\
\hline No & $7883(81.1)$ & \\
\hline Parent Age, Mean (SD) & $39.92(6.39)$ & $23.00-80.00$ \\
\hline Parent Marital Status, N (\%) & & \\
\hline Married & $6482(67.3)$ & \\
\hline Not Married & $3154(32.7)$ & \\
\hline Parents Living Together, N (\%) & & \\
\hline Yes & $7064(73.3)$ & \\
\hline No & $2572(26.7)$ & \\
\hline Parent Education & & \\
\hline$<$ High School Diploma & $492(5.1)$ & \\
\hline $\begin{array}{l}\text { High School Diploma or } \\
\text { Equivalent }\end{array}$ & $946(9.7)$ & \\
\hline Some College & $2539(26.2)$ & \\
\hline Bachelor's Degree & $2408(24.8)$ & \\
\hline Graduate Degree & $3323(34.2)$ & \\
\hline Income, $\mathrm{N}(\%)$ & & \\
\hline$<\$ 50,000$ & $2695(30.4)$ & \\
\hline$\$ 50,000-\$ 100,000$ & 3664 (41.3) & \\
\hline$>\$ 100,000$ & $2515(28.3)$ & \\
\hline
\end{tabular}




\begin{tabular}{|c|c|c|}
\hline $\begin{array}{l}\text { Area Deprivation Index (Income } \\
\text { Disnarity) }\end{array}$ & $2.17(1.35)$ & $-1.13-8.16$ \\
\hline $\begin{array}{l}\text { Basic Needs Unaffordability } \\
\text { (PhenX) }\end{array}$ & $0.48(1.10)$ & $0.00-7.00$ \\
\hline Area Number of Crimes & $54512.12(87439.22)$ & $0.00-348049.30$ \\
\hline $\begin{array}{l}\text { Neighborhood Crime and Safety } \\
\text { (PhenX) }\end{array}$ & $3.87(0.98)$ & $1.00-5.00$ \\
\hline $\begin{array}{l}\text { Youth Access to Substances } \\
\text { (CRPF) }\end{array}$ & $9.52(3.14)$ & $1.00-30.00$ \\
\hline Trauma Exposure (KSADs) & $0.53(1.10)$ & $0.00-17.00$ \\
\hline \multicolumn{3}{|c|}{ Parent Mental Health and Familial Variables, M (SD) } \\
\hline \multicolumn{3}{|l|}{ Parent Report } \\
\hline \multicolumn{3}{|l|}{ ASR } \\
\hline Externalizing Behaviors & $45.94(9.67)$ & $30.00-90.00$ \\
\hline Internalizing Symptoms & $48.21(10.57)$ & $30.00-95.00$ \\
\hline Personal Strength & $47.87(9.37)$ & $20.00-60.00$ \\
\hline $\begin{array}{l}\text { Parent Alcohol Use, days } \\
\text { intoxicated in past } 6 \\
\text { months }\end{array}$ & $1.37(6.34)$ & $0.00-180.00$ \\
\hline $\begin{array}{l}\text { Parent Nicotine Use, times } \\
\text { per day in past } 6 \text { months }\end{array}$ & $1.01(4.50)$ & $0.00-100.00$ \\
\hline $\begin{array}{l}\text { Parent Drug Use, days in } \\
\text { past } 6 \text { months }\end{array}$ & $1.97(14.85)$ & $0.00-180.00$ \\
\hline Family History (FHAM-S) & $2.36(2.02)$ & $0.00-8.00$ \\
\hline Family Conflict (FES) & $2.52(1.95)$ & $0.00-9.00$ \\
\hline \multicolumn{3}{|l|}{ Youth Report } \\
\hline Family Conflict (FES) & $2.02(1.96)$ & $0.00-9.00$ \\
\hline Parental Monitoring (PMQ) & $4.39(0.51)$ & $1.00-5.00$ \\
\hline $\begin{array}{l}\text { Study Caregiver Acceptance } \\
\text { (CRPBI) }\end{array}$ & $2.78(0.30)$ & $1.00-3.00$ \\
\hline $\begin{array}{l}\text { Secondary Caregiver } \\
\text { Acceptance (CRPBI) }\end{array}$ & $2.69(0.38)$ & $1.00-3.00$ \\
\hline \multicolumn{3}{|c|}{ Youth Mental Health Symptoms and Behaviors, M (SD) } \\
\hline \multicolumn{3}{|l|}{$\mathrm{CBCL}$} \\
\hline Externalizing Behaviors & $45.89(10.36)$ & $33.00-84.00$ \\
\hline Internalizing Symptoms & $48.75(10.68)$ & $33.00-93.00$ \\
\hline Mania Symptoms (PGBI) & $1.37(2.84)$ & $0.00-28.00$ \\
\hline \multicolumn{3}{|l|}{ Youth Report } \\
\hline \multicolumn{3}{|l|}{ BIS/BAS } \\
\hline Drive & $4.18(3.08)$ & $0.00-12.00$ \\
\hline Fun Seeking & $5.74(2.66)$ & $0.00-12.00$ \\
\hline
\end{tabular}




\begin{tabular}{|c|c|c|}
\hline Reward Responsiveness & $8.85(2.38)$ & $0.00-12.00$ \\
\hline Inhibition & $5.53(2.85)$ & $0.00-12.00$ \\
\hline \multicolumn{3}{|l|}{ UPPS-P } \\
\hline Lack of Perseverance & $7.06(2.26)$ & $4.00-16.00$ \\
\hline Lack of Planning & $7.73(2.37)$ & $4.00-16.00$ \\
\hline Negative Urgency & $8.50(2.65)$ & $4.00-16.00$ \\
\hline Positive Urgency & $7.98(2.95)$ & $4.00-16.00$ \\
\hline Sensation Seeking & $9.79(2.68)$ & $4.00-16.00$ \\
\hline \multicolumn{3}{|l|}{ Modifiable Risks, M (SD) } \\
\hline Body Mass Index & $18.81(4.00)$ & $11.10-35.87$ \\
\hline Waist Circumference, Inches & $26.68(4.95)$ & $0.00-90.00$ \\
\hline \multicolumn{3}{|l|}{ Parent Report } \\
\hline $\begin{array}{l}\text { Extracurricular Involvement, } \\
\text { hours } \\
\text { per week (SAIQ) }\end{array}$ & $17.99(13.84)$ & $0.00-170.00$ \\
\hline Art & $5.07(6.32)$ & $0.00-54.00$ \\
\hline Individual Sports & $4.93(5.7)$ & $0.00-70.00$ \\
\hline Team Sports & $8.06(7.65)$ & $0.00-54.00$ \\
\hline \multicolumn{3}{|l|}{ Sleep (SDSC) } \\
\hline Amount, Hours, N (\%) & & . \\
\hline$<5$ & $24(0.25)$ & \\
\hline $5-7$ & $305(3.14)$ & \\
\hline $7-8$ & $1197(12.32)$ & \\
\hline $8-9$ & $3615(37.21)$ & \\
\hline $9-11$ & 4575 (47.09) & \\
\hline \multicolumn{2}{|l|}{ Initiation, Minutes, N (\%) } & . \\
\hline$<15$ & $3760(38.70)$ & \\
\hline $15-30$ & $3950(40.65)$ & \\
\hline $30-45$ & $1209(12.44)$ & \\
\hline $45-60$ & $534(5.50)$ & \\
\hline$>60$ & $263(2.71)$ & \\
\hline \multicolumn{3}{|l|}{ Youth Report } \\
\hline $\begin{array}{l}\text { Physical Activity, Days per } \\
\text { Week }\end{array}$ & $3.49(2.32)$ & $0.00-7.00$ \\
\hline \multicolumn{3}{|l|}{$\begin{array}{l}\text { Screen Media Activity, Hours } \\
\text { (STQ) }\end{array}$} \\
\hline Weekday & $2.57(2.61)$ & $0.00-24.00$ \\
\hline Social Network Sites & $0.11(0.42)$ & $0.00-4.00$ \\
\hline $\begin{array}{l}\text { Text and Instant } \\
\text { Messages }\end{array}$ & $0.22(0.56)$ & $0.00-4.00$ \\
\hline TV shows or Movies & $1.11(1.10)$ & $0.00-4.00$ \\
\hline
\end{tabular}




$\begin{array}{cll}\text { R-rated Movies } & 0.40(0.73) & 0.00-4.00 \\ \text { Videos (e.g, YouTube) } & 0.93(1.17) & 0.00-4.00 \\ \text { Video Games } & 0.92(1.14) & 0.00-4.00 \\ \text { Mature-rated Games } & 0.63(1.06) & 0.00-4.00 \\ \text { Video Chat } & 0.18(0.50) & 0.00-4.00 \\ \text { Weekend } & 4.00(2.65) & 0.00-24.00 \\ \text { Social Network Sites } & 0.13(0.54) & 0.00-4.00 \\ \text { Text and Instant } & 0.26(0.71) & 0.00-4.00 \\ \text { Messages } & 1.75(1.27) & 0.00-4.00 \\ \text { TV shows or Movies } & 1.31(1.38) & 0.00-4.00 \\ \text { Videos (e.g, YouTube) } & 1.40(1.35) & 0.00-4.00 \\ \text { Video Games } & 0.23(0.67) & 0.00-4.00 \\ \text { Video Chat } & 0.40(0.73) & 0.00-4.00 \\ \text { R-rated Movies } & 0.63(1.06) & 0.00-4.00 \\ \text { Mature-rated Games } & \end{array}$

Abbreviations: ASR, Adult Self-Report; BIS/BAS, Behavioral Inhibition/Behavioral Activation Scale; CBCL, Child Behavior Checklist; CRPBI, Child's Report of Parental Behavior Inventory - Acceptance subscale; CRPF, Community Risk and Protective Factors; FES, Family Environment Scale - Family Conflict subscale; FHAM-S, Family History Assessment Module Screener; KSADS, Kiddie Schedule for Affective Disorders and Schizophrenia; PGBI, Parent General Behavior Inventory for Children and Adolescents - Mania subscale; PMQ, Parental Monitoring Questionnaire; SAIQ, Sports and Activities Involvement Questionnaire; SDSC, Sleep Disturbance Scale for Children; STQ, Screen Time Questionnaire; UPPS-P, Impulsive Behavior Scale. 
Table 2. Variables with univariate correlation (Spearman $r$ and P-value FDR corrected) with age-corrected fluid cognitive functioning and variable importance (VI) in the stacked model. Order of variables are in alphabetic order.

\begin{tabular}{|c|c|c|c|}
\hline \multirow[b]{2}{*}{ Variable } & \multicolumn{3}{|c|}{ Baseline } \\
\hline & $\mathrm{r}$ & $\mathrm{pFDR}$ & VI \\
\hline ABCD Site & 0.018 & 0.09 & 2.960 \\
\hline Area Deprivation Index (Income & & & \\
\hline Disparity) & -0.242 & $<0.001$ & 78.541 \\
\hline Area Number of Crimes & -0.003 & 0.78 & 3.301 \\
\hline ASR: Externalizing Behaviors & -0.019 & 0.07 & 2.909 \\
\hline ASR: Internalizing Symptoms & -0.039 & $<0.001$ & 3.694 \\
\hline ASR: Parent Alcohol Use & 0.020 & 0.06 & 0.963 \\
\hline ASR: Personal Strength & 0.118 & $<0.001$ & 17.292 \\
\hline $\begin{array}{l}\text { Basic Needs Unaffordability } \\
\text { (PhenX) }\end{array}$ & -0.163 & $<0.001$ & 26.448 \\
\hline BIS/BAS: Drive & -0.144 & $<0.001$ & 32.518 \\
\hline BIS/BAS: Fun Seeking & -0.067 & $<0.001$ & 5.085 \\
\hline BIS/BAS: Inhibition & -0.024 & 0.03 & 1.926 \\
\hline BIS/BAS: Reward Responsiveness & -0.052 & $<0.001$ & 4.713 \\
\hline Body Mass Index & -0.111 & $<0.001$ & 17.485 \\
\hline CBCL: Externalizing Behaviors & -0.126 & $<0.001$ & 23.911 \\
\hline CBCL: Internalizing Symptoms & -0.050 & $<0.001$ & 4.332 \\
\hline $\begin{array}{l}\text { Extracurricular Involvement } \\
\text { (SAIQ) }\end{array}$ & 0.212 & $<0.001$ & 64.142 \\
\hline Family Conflict (FES) & -0.105 & $<0.001$ & 14.686 \\
\hline Family History (FHAM-S) & 0.012 & 0.25 & 2.398 \\
\hline Family Relationships (FES) & 0.013 & 0.23 & 0.766 \\
\hline Gender & 0.039 & $<0.001$ & 4.818 \\
\hline Income $<\$ 50,000$ & -0.260 & $<0.001$ & 98.740 \\
\hline Income $\$ 50,000$ - $\$ 100,000$ & 0.030 & 0.004 & 3.929 \\
\hline Mania Symptoms (PGBI) & -0.147 & $<0.001$ & 27.484 \\
\hline $\begin{array}{l}\text { Neighborhood Crime and Safety } \\
(\text { PhenX) }\end{array}$ & 0.157 & $<0.001$ & 25.169 \\
\hline Parent Age & 0.118 & $<0.001$ & 14.430 \\
\hline Parent Education: Bachelor's & & & \\
\hline Degree & 0.066 & $<0.001$ & 6.928 \\
\hline Parent Education: Some College & -0.129 & $<0.001$ & 21.737 \\
\hline Parent Marital Status & 0.201 & $<0.001$ & 45.079 \\
\hline Parental Conflict (FES) & -0.049 & $<0.001$ & 3.331 \\
\hline Parental Monitoring (PMQ) & 0.067 & $<0.001$ & 6.884 \\
\hline Parents Living Together & 0.176 & $<0.001$ & 32.177 \\
\hline Physical Activity (SAIQ) & 0.093 & $<0.001$ & 10.502 \\
\hline
\end{tabular}


Racial Minority Status

Screen Media Activity: Weekday

(STQ)

Screen Media Activity: Weekend (STQ)

Secondary Caregiver Acceptance

(CRPBI)

Sleep Amount (SDSC)

Sleep Initiation (SDSC)

Study Caregiver Acceptance

(CRPBI)

Trauma Exposure (KSADs)

UPPS: Lack of Perseverance

UPPS: Lack of Planning

UPPS: Negative Urgency

UPPS: Positive Urgency

UPPS: Sensation Seeking

Waist Circumference

Youth Access to Substances

(CRPF)

Abbreviations: ABCD, Adolescent Brain Cognitive

BIS/BAS, Behavioral Avoidance/Inhibition Scales; CBCL, Child Behavior Checklist; CRPBI, Child's Report of Parental Behavior Inventory - Acceptance subscale; CRPF, Community Risk and Protective Factors; FES, Family Environment Scale; FHAM-S, Family History Assessment Module Screener; KSADs, Kiddie Schedule for Affective Disorders and Schizophrenia; PGBI, Parent General Behavior Inventory for Children and Adolescents - Mania subscale; PMQ, Parental Monitoring Scale; SAIQ, Sports and Activities Involvement Questionnaire; SDSC, Sleep Disturbance Scale for Children; STQ, Screen Time Questionnaire; UPPS-P, Impulsive Behavior Scale.

\begin{tabular}{rrr}
-0.196 & $<0.001$ & 48.963 \\
-0.148 & $<0.001$ & 26.642 \\
-0.089 & $<0.001$ & 9.183 \\
& & \\
0.005 & 0.67 & 0.866 \\
0.168 & $<0.001$ & 31.073 \\
-0.045 & 0.000 & 2.968 \\
0.019 & & \\
-0.054 & 0.07 & 2.378 \\
-0.092 & $<0.001$ & 3.591 \\
0.002 & $<0.001$ & 21.918 \\
-0.068 & 0.85 & 4.000 \\
-0.133 & $<0.001$ & 5.669 \\
0.023 & $<0.001$ & 25.419 \\
-0.084 & 0.03 & 2.103 \\
0.073 & $<0.001$ & 7.706 \\
& & 8.670 \\
\hline
\end{tabular}

\section{(1)}




\section{FIGURES}

Figure 1. Variable importance (VI) for models predicting Fluid Cognitive Functioning in the ABCD cohort $(n=9,718)$. Variable importance $(\mathrm{VI})$ is based on the stacked ensemble. Variables with orange or blue coloring have a negative or positive univariate correlation with Fluid Cognitive Functioning, respectively. Error bars represent the $95 \%$ confidence interval, taken across partitions. The accompanying pie chart depicts percent of variance explained by the model and its 95\% confidence interval. Abbreviations: BIS/BAS, Behavioral Inhibition/Behavioral Activation Scale; CBCL, Child Behavior Checklist UPPS-P, Impulsive Behavior Scale.

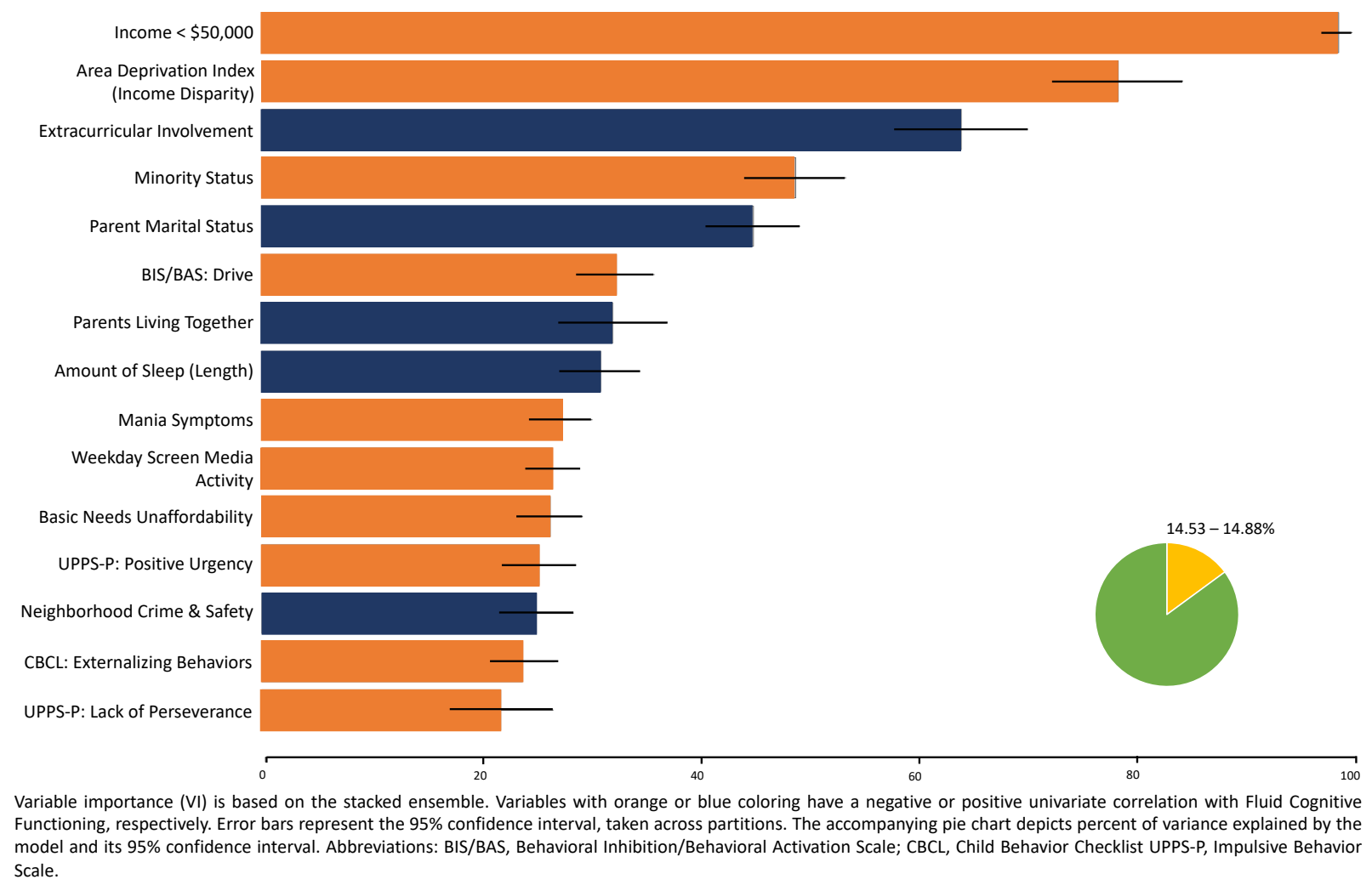


Figure 2. Partial dependence plots showing the marginal effect each of the 15 variables with the highest importance has on age corrected fluid cognitive functioning scores. Abbreviations: BIS/BAS, Behavioral Avoidance/Inhibition Scales; CBCL, Child Behavior Checklist.
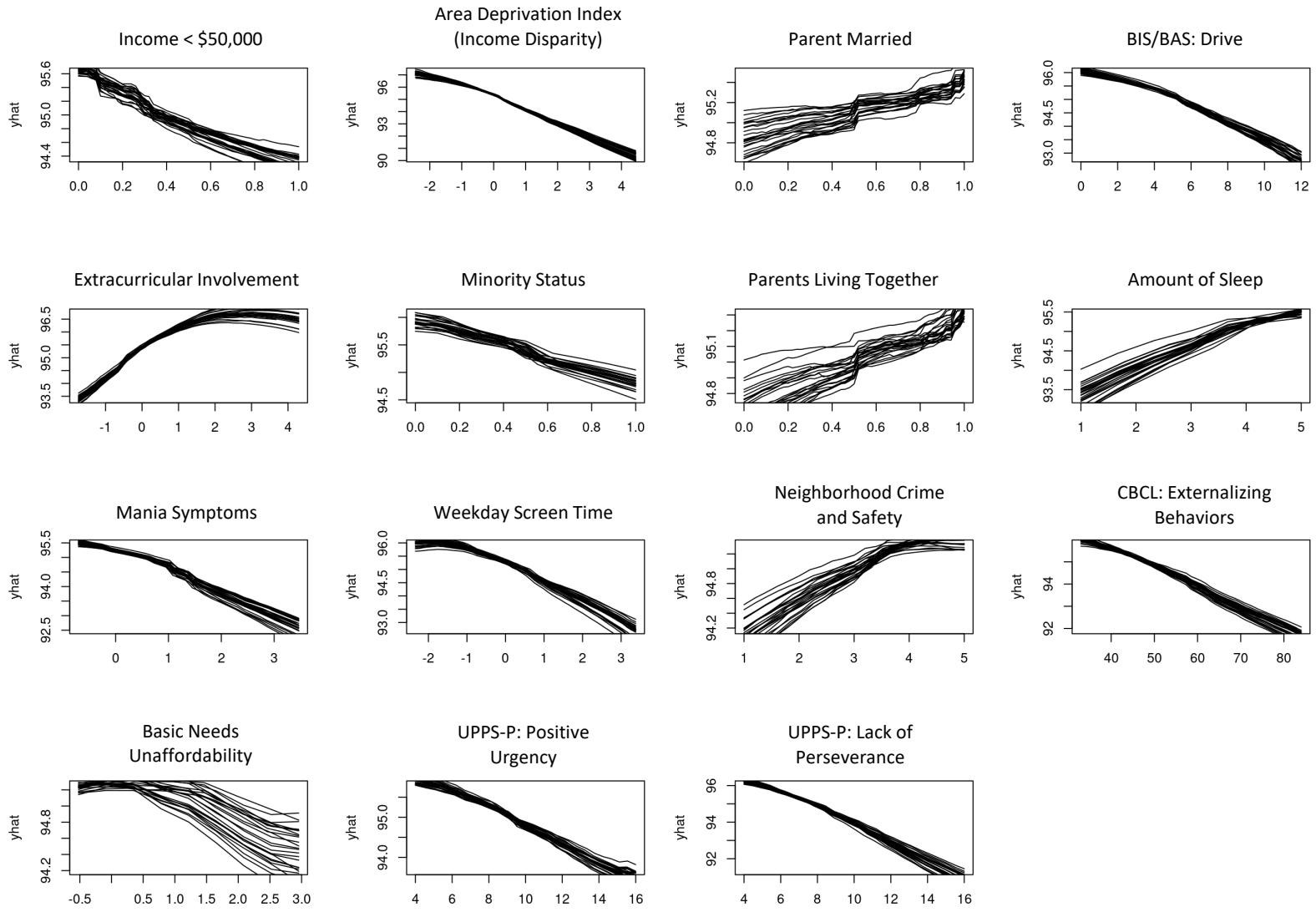

Abbreviations: BIS/BAS, Behavioral Avoidance/Inhibition Scales; CBCL, Child Behavior Checklist. 


\section{Supplementary Information}

Title: Extracurricular activities, screen media activity, and sleep may be modifiable factors influencing children's cognitive functioning: Evidence from the ABCD study

Authors: Namik Kirlic, $\mathrm{PhD}^{1}$, Janna M. Colaizzi, $\mathrm{PhD}^{1}$, Kelly T. Cosgrove, $\mathrm{MA}^{1}$, Zsofia P. Cohen ${ }^{1}$, Hung-Wen Yeh, $\mathrm{PhD}^{1,2}$, Florence Breslin, $\mathrm{MS}^{1}$, Amanda Sheffield Morris, $\mathrm{PhD}^{1,3}$, Robin Aupperle, $\mathrm{PhD}^{1,4}$, and Martin P. Paulus, $\mathrm{MD}^{1}$

Affiliations: ${ }^{1}$ Laureate Institute for Brain Research, Tulsa, OK, USA; ${ }^{2}$ Health Services \& Outcomes Research, Children's Mercy Hospital, Kansas City, MO; ${ }^{3}$ Department of Human Development and Family Sciences, Oklahoma State University, Tulsa, OK; ${ }^{4}$ School of Community Medicine, University of Tulsa, Tulsa, OK

Corresponding Author: Namik Kirlic, PhD, Laureate Institute for Brain Research, 6655 South Yale Ave. Tulsa, OK 74136. nkirlic@laureateinstitute.org; Phone: (918) 502-5747 


\section{$\underline{\text { Supplementary Materials }}$}

Overview

1. Supplementary methods

a. Measures

b. Machine learning methods

2. Supplementary tables

a. Table S1. Variables included in the machine learning analysis.

3. Supplementary figures

a. Figure S1. The process of repeated nested CV.

b. Figure S2. The inner-loop of repeated nested CV

c. Figure S3. Distribution of age-corrected Fluid Cognitive Functioning (FCF) composite scores.

d. Figure S4. Percent of variance explained for age corrected fluid cognitive functioning score by 47 demographic, psychological, and environmental predictors $(\mathrm{n}=9,719)$.

e. Figure S5. Scatter plots between potentially modifiable variables and Fluid Cognitive Fucntioning composite scores. 


\section{Supplementary methods}

a. Measures

Family Demographics. Primary caregivers provided demographic information regarding the parent/guardian, their partner, the youth's grandparents, and the youth using the PhenX survey toolkit (D. M. Barch et al., 2018; P. J. Stover, W. R. Harlan, J. A. Hammond, T. Hendershot, \& C. M. Hamilton, 2010).

Mental Health and Psychopathology. Behavioral inhibition and activation were assessed using the Behavioral Inhibition \& Behavioral Activation Scales (BIS/BAS) from PhenX (D. M. Barch et al., 2018; Pagliaccio et al., 2016) with subscales for drive (intensity of goal-directed behavior), fun seeking (enjoyment for its own sake; spontaneity), and reward responsiveness (excitement over reinforcing outcomes), as well as inhibition (worry, fearfulness). Impulsivity was measured via the UPPS-P for Children from PhenX (D. M. Barch et al., 2018; Lynam, 2013; Zapolski, Stairs, Settles, Combs, \& Smith, 2010), with four subscales including lack of premeditation, positive and negative urgency (difficulty controlling emotions), sensation seeking, and lack of perseverance.

Dimensional psychopathology and adaptive functioning were measured using the Achenbach Child Behavior Checklist (CBCL; T.M. Achenbach, 2009; D. M. Barch et al., 2018), normed for sex, age, and ethnicity. We utilized the broader syndrome subscale scores for internalizing and externalizing symptoms. Mania symptoms were measured using a sum score from the mania subscale of the Parent General Behavior Inventory for Children and Adolescents (D. M. Barch et al., 2018; E. A. Youngstrom, T. W. Frazier, C. Demeter, J. R. Calabrese, \& R. L. 
Findling, 2008). Finally, exposure to traumatic events, including the death of a loved one, or the youth witnessing or experiencing physical or sexual assault, domestic violence, or other violent acts was assessed with questions from the Kiddie Schedule for Affective Disorders and Schizophrenia for DSM-5 (KSADS-5; D. M. Barch et al., 2018; Kobak \& Kaufman, 2015).

Physical Health and Extracurricular Engagement. Youth standardized measures of height, weight, body mass index (BMI), and waist circumference were measured using the National Health and Nutrition Examination Survey (CDC (Division of Nutrition, 2016). Parents reported on the past and current involvement of youth in sports, activities, music, or hobbies using the Sports and Activities Involvement Questionnaire (modified from the Vermont Health Behavior Questionnaire; Huppertz et al., 2016). Youth also self-reported their level of physical exercise via items from the Youth Risk Behavior Survey (YBR; CDC, 2016b). Youth sleep initiation delays and average total sleep length were assessed using the parent-report Sleep Disturbances Scale for Children (O. Bruni et al., 1996). Finally, youth self-reported the estimated amount of time typically spent on multiple types of screen media activity (e.g., watching TV, movies, or internet videos and active/social use such as playing video games, texting, or visiting social media sites) on a typical day week or weekend day (K. S. Bagot et al., 2018). Questions were also asked relating to time spent engaging with mature rated movies and video games.

Environmental Adversity. To address influences on socioeconomic status other than income alone, we used a measure of relative income disparity based on residential histories and measures of economic insecurity and neighborhood safety. For relative income disparity, we used the area deprivation index (ADI; Singh (2003)), a composite measure of neighborhood socioeconomic 
disadvantage. ADI uses US Census data related to poverty, education, housing, and employment within a given zip code to create census-based regions for neighborhood risk. A grand total of neighborhood crime rates was also included with various forms of violent and non-violent reported crimes based on US Census data (K. S. Bagot et al., 2018). Economic insecurity was measured using the best practices for conceptualizing and measuring social class in psychological research outlined in Diemer et al. (2012). One's ability to pay for basic needs such as medical care, phone service, utilities, or food were summed and used in analyses. Finally, a sum score of parent-reported questions relating to the perceived safety of and presence of crime in one's neighborhood including feeling safe walking alone and level of violence and crime (ranging from "strongly agree" to "strongly disagree") was derived from the Safety from Crime subscale of the PhenX Toolkit (Toolkit, 2016; R. A. Zucker et al., 2018).

In addition to markers of environmental adversity, we also included measures addressing the family environment. The Family Conflict subscale of the Family Environment Scale (Moos \& Moos, 1994; R. A. Zucker et al., 2018) was completed by both the parent and the youth. Items included questions regarding family members losing their temper, arguing, or acting violent with higher scores indicating more conflict. Parents self-reported dimensional psychopathology and adaptive functioning using the Achenbach Adult Self Report Questionnaire (ASR; T.M. Achenbach, 2009; D. M. Barch et al., 2018), from which items regarding parent substance use, personal strength, and internalizing and externalizing symptoms were used. Finally, we included questions relating to history of psychopathology among first- and second-degree relatives (i.e., substance use, depression, mania, psychosis, and antisocial personality disorder) from the Family History Assessment Module Screener (FHAM-S; D. M. Barch et al., 2018; J. P. Rice et al., 1995). Youth also reported on their perception of parental monitoring using the Parental 
Monitoring Scale, including parent/child contact, frequency of family dinners, parents knowing child's whereabouts, and child disclosure to parents (H. D. Chilcoat \& J. C. Anthony, 1996; R. A. Zucker et al., 2018) and their perception of parental warmth, acceptance, and responsiveness via the Acceptance Subscale of the Child Report of Behavior Inventory modified for ABCD to address primary and secondary caregivers' behaviors (Schaefer, 1965; R. A. Zucker et al., 2018). Finally, parent-reported youth accessibility to substances was measured using the Community Risk and Protective Factors scale from the PhenX toolkit (Arthur et al., 2007; K. M. Lisdahl et al., 2018).

\section{b. Machine learning methods}

Selection of algorithms. In this work, we implemented four algorithms: elastic net (ENET) (Barsaglini, Sartori, Benetti, Pettersson-Yeo, \& Mechelli), support vector regression (SVR) (Suykens \& Vandewalle, 1999), conditional random forest (CRF) (Hothorn, Hornik, \& Zeileis, 2006), and k-Nearest Neighbors (knn) (Keller, Gray, \& Givens, 1985).

Repeated nested cross-validation. It is well known that using the same dataset to build a prediction model and evaluate its performance can cause overestimation in the model performance (Varma \& Simon, 2006). If we have two datasets, one of them can be used to train a prediction model, and the other to evaluate the model performance. Because we had only one dataset, one possibility was to split the data into a training set for model building and a validation 
set for model evaluation. Alternatively, the whole dataset could be partitioned to " $K$ " parts, where one part served as a validation set and the other parts were combined to serve as a training set, and the process iterated so that each part served as a validation set exactly once (Outer loop). For a given machine learning method, a training set could be partitioned into $K$ parts and the optimal tuning parameters could be determined by $K$-fold cross validation (Inner loop). The trained model was then applied to make predictions in the corresponding validation set. Iterating across the " $K$ " parts, the " $K$ " sets of predicted values were combined to compare with the observed values to evaluate model performance. The whole procedure is known as nested or double cross-validation (Stone, 1974; Varma \& Simon, 2006) and was repeated by different partition indices to assess the variability of the results (Filzmoser, Liebmann, \& Varmuza, 2009). We chose to repeat 100 times nested cross-validation (CV), with 5-fold CV for both the outer and the inner loops.

\section{Stacked regression/Super learner (the inner loop)}

In the inner loop, we applied four machine learning methods and then combined their predictions by stack ensemble. Suppose a training set $\mathbf{D}$ was expressed as a matrix, concatenated in columns by a response vector $\boldsymbol{y}$ and a predictor matrix $\mathbf{X}$, with $n \mathrm{X}\left(K_{1}-1\right) / K_{1}$ observations.

Step 1: Building base learners. Each base learner was built by $K_{2}$-fold CV, which consisted of data partition and parameter optimization.

Data partition: A training set $D$ was partitioned into $K_{2}$ non-overlapped parts where $\left(K_{2}-\right.$ 1) parts were used to train a model with hyper-parameter $\boldsymbol{\theta}_{j}$, which was then used to 
predict the held-out part and to compute the corresponding model performance metric $\left(R^{2}\right.$ here); this process was repeated so that this step would give $K_{2}$ values of $R^{2}$.

Optimization: For a grid of $J$ hyper-parameter combinations, denoted as $\boldsymbol{\theta}_{\mathrm{j}}, j=1, \ldots, J$, the same partition indices of $K_{2}$-fold CV were applied to each $\boldsymbol{\theta}_{j}$. Note $\boldsymbol{\theta}_{j}$ was either a vector (two parameters for eNet) or a scalar (other methods). The optimal hyperparameter $\boldsymbol{\theta}_{\mathrm{Opt}}$ were determined by the "one-standard-error" (1-SE) rule: among the $\boldsymbol{\theta}_{\mathrm{j}}$ 's whose mean $R^{2}$ (averaged across $K_{1}$ test sets) fell within one SE of the maximal mean $R^{2}$, the $\boldsymbol{\theta}_{\mathrm{j}}$ that corresponded to the most parsimonious model was $\boldsymbol{\theta}_{\text {Opt. Note that }}$ SE of the metric is defined as

$$
S E=\frac{S D}{\sqrt{K_{2}}}
$$

Should $\boldsymbol{\theta}_{\text {Opt }}$ be identified, the predicted values of the held-out sets at $\boldsymbol{\theta}_{\text {Opt, }}$ denoted as $\widehat{\boldsymbol{y}}\left(\boldsymbol{\theta}_{\mathrm{Opt}}\right)$ were extracted for stack ensemble.

Step 2: Building a stack ensemble model. The predicted values of the optimal hyperparameter $\boldsymbol{\theta}_{\text {Opt }}$ from $L$ base learners (Step 1) were then combined with the observed response values into a matrix $\widehat{\mathbf{D}}$ with $(L+1)$ columns. The observed response could be regressed on the $m$ variables to obtain a stack ensemble model $\mathrm{M}^{\mathrm{S}}$. We chose to average predicted values of base learners weighted by their mean $R^{2}$ values from Step 1 .

Hyper-parameter selection. Elastic net had two tuning parameters $\alpha$ and $\lambda$. CRF was tuned on a single parameter "mtry" for the number of predictors randomly selected to grow a tree; the 
number of trees was set at 500 and not tuned. Although in theory SVR may have three parameters: cost, $\epsilon$, and $\sigma$, the scale parameter $\sigma$ ("sigma") for radial basis function was estimated by the midpoint of the 10th and 90th percentiles of Euclidean distance between all training points (Caputo, Sim, Furesjo, \& Smola, 2002); also, the cost and $\epsilon$ parameters have some relationship and Kuhn (2014) suggests using cost (“C”) as the only tuning parameter. The knn algorithm had a single tuning parameter $k$, the number of nearest neighbors. To alleviate computation burden, we used random search (Bergstra \& Bengio, 2012) with adaptive resampling algorithm (Kuhn, 2014) for no more than 15 parameter combinations, so the actually used parameter combinations and values varied with replications and training sets. 


\section{Supplementary tables}

Table S1. Variables included in the machine learning analysis.

\begin{tabular}{c} 
Demographics (Deanna M Barch et al., 2018; Patrick J Stover, William R Harlan, Jane A \\
Hammond, Tabitha Hendershot, \& Carol M Hamilton, 2010) \\
\hline$\cdot$ Age \\
$\cdot$ Gender \\
$\cdot$ Racial Minority Status \\
$\cdot$ Twin and Sibling Status
\end{tabular}

\section{Environment}

- Income Disparity and Affordability (Patrick J Stover et al., 2010)

- Youth Access to Substances - CRPF

(Arthur et al., 2007; Krista M Lisdahl et al., 2018)
- Neighborhood Crime and Safety (Deanna M

Barch et al., 2018)

- Trauma - KSADs (Puig-Antich \& Ryan, 1986)

\section{Parent Mental Health and Familial Variables}

- Psychopathology and Substance Use -

ASR (Thomas M Achenbach, 1997) Family Conflict - FES (Moos \& Moos, 1994; Robert A Zucker et al., 2018)

- Caregiver Acceptance - CRBPI (Schaefer, · Family History - FHAM-S (John P Rice et

- Parental Monitoring - PMQ (Howard D

Chilcoat \& James C Anthony, 1996) and 1965; Robert A Zucker et al., 2018) al., 1995)

\section{Youth Mental Health Symtpoms and Behaviors}


· Pscyhopathology - CBCL (Thomas M

Achenbach \& Ruffle, 2000)
- Mania Symptoms - PGBI (Eric A

Youngstrom, Thomas W Frazier, Christine

Demeter, Joseph R Calabrese, \& Robert L

Findling, 2008)

· Behavioral Avoidance and Inhibition - $\quad$ Impulsivity - UPPS-P (Whiteside, Lynam, BIS/BAS (Carver \& White, 1994) Miller, \& Reynolds, 2005)

\section{Modifiable Risks}

- BMI And Waist Circumference (CDC · Sleep Quality - SDSC (Oliviero Bruni et al., (Division of Nutrition, 2016)

· Extracurricuar Involvement - SAIQ $\quad$ Physical Activity - YRB (CDC, 2016a)

(Huppertz et al., 2016)

- Screen Media Activity - STQ (K. Bagot et al., 2018)

Abbreviations: ASR, Adult Self-Report; BIS/BAS, Behavioral Avoidance/Inhibition Scales; BMI, Body Mass Index; CBCL, Child Behavior Checklist; CRPBI, Child's Report of Parental Behavior Inventory - Acceptance subscale; CRPF, Community Risk and Protective Factors; FES, Family Environment Scale - Family Conflict Subscale; FHAM-S, Family History Assessment Module Screener; KSADs, Kiddie Schedule for Affective Disorders and Schizophrenia for DSM-5; PGBI, Parent General Behavior Inventory for Children and Adolescents - Mania subscale; PMQ, Parental Monitoring Questionnaire; SAIQ, Sports and Activities Involvement Questionnaire; SDSC, Sleep Disturbance Scale for Children; STQ , Screen Time Questionnaire; UPPS-P, Impulsive Behavior Scale; YRB, Youth Risk Behavior Survey. 



\section{Supplementary figures}

\section{Repeated nested cross-validation}

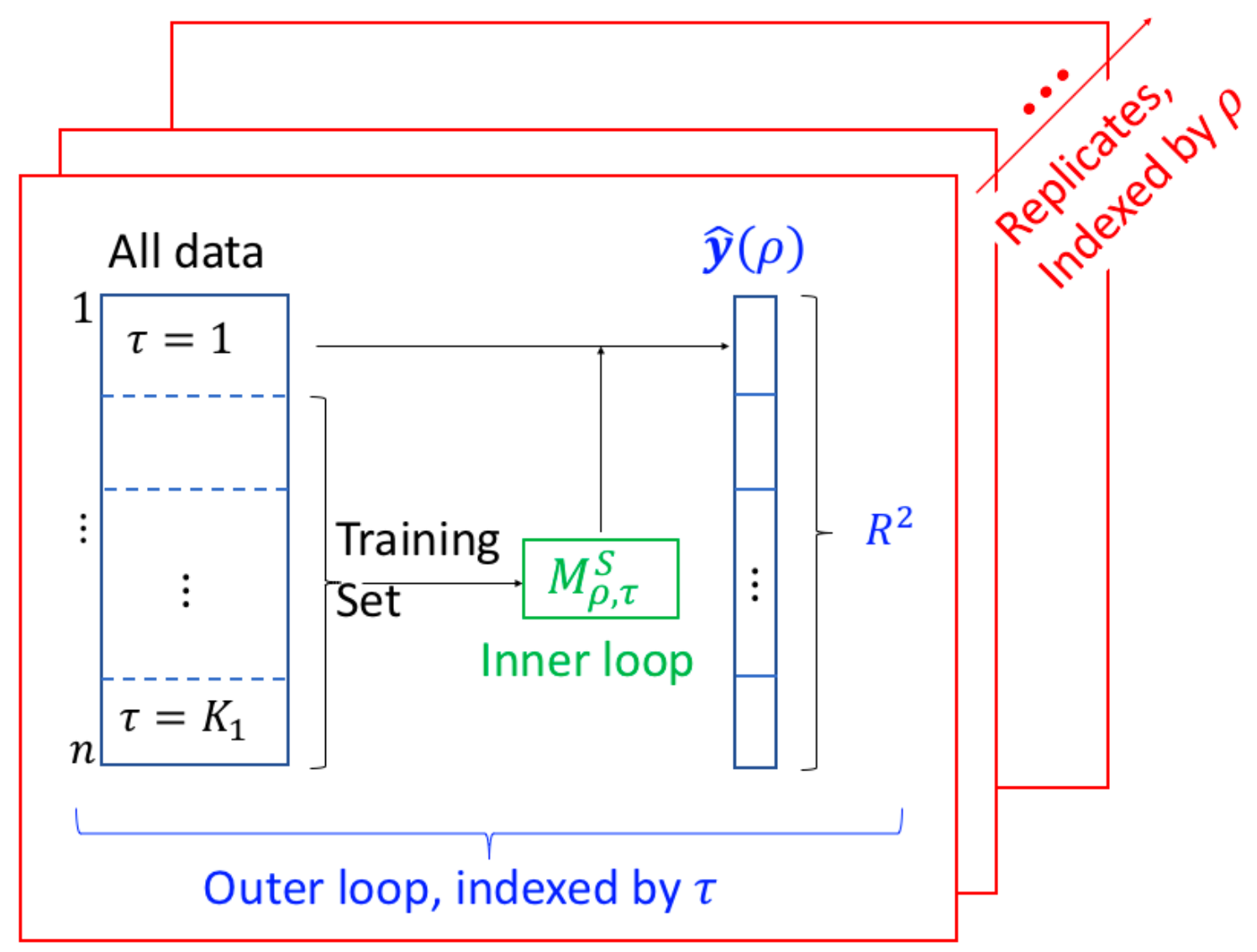

Figure S1. The process of repeated nested CV. This figure illustrates the process of nested $\mathrm{CV}$ repeated 100 times indexed by $\rho$. In the outer loop, the original data of $n$ subjects were partitioned into $K_{1}$ parts indexed by $\tau$. For each iterate, $\left(K_{1}-1\right)$ parts served as a training set to optimize tuning parameters in the inner loop (see Supplementary Figure S1b.). The stack model $\mathrm{M}_{\rho, \tau}^{\mathrm{s}}$ from the inner loop was then used to predict the held-out set of the outer loop. Predicted values $\widehat{\boldsymbol{y}}(\rho)$ were combined across held-out sets to evaluate the performance of the stack models. 


\section{Inner loop}

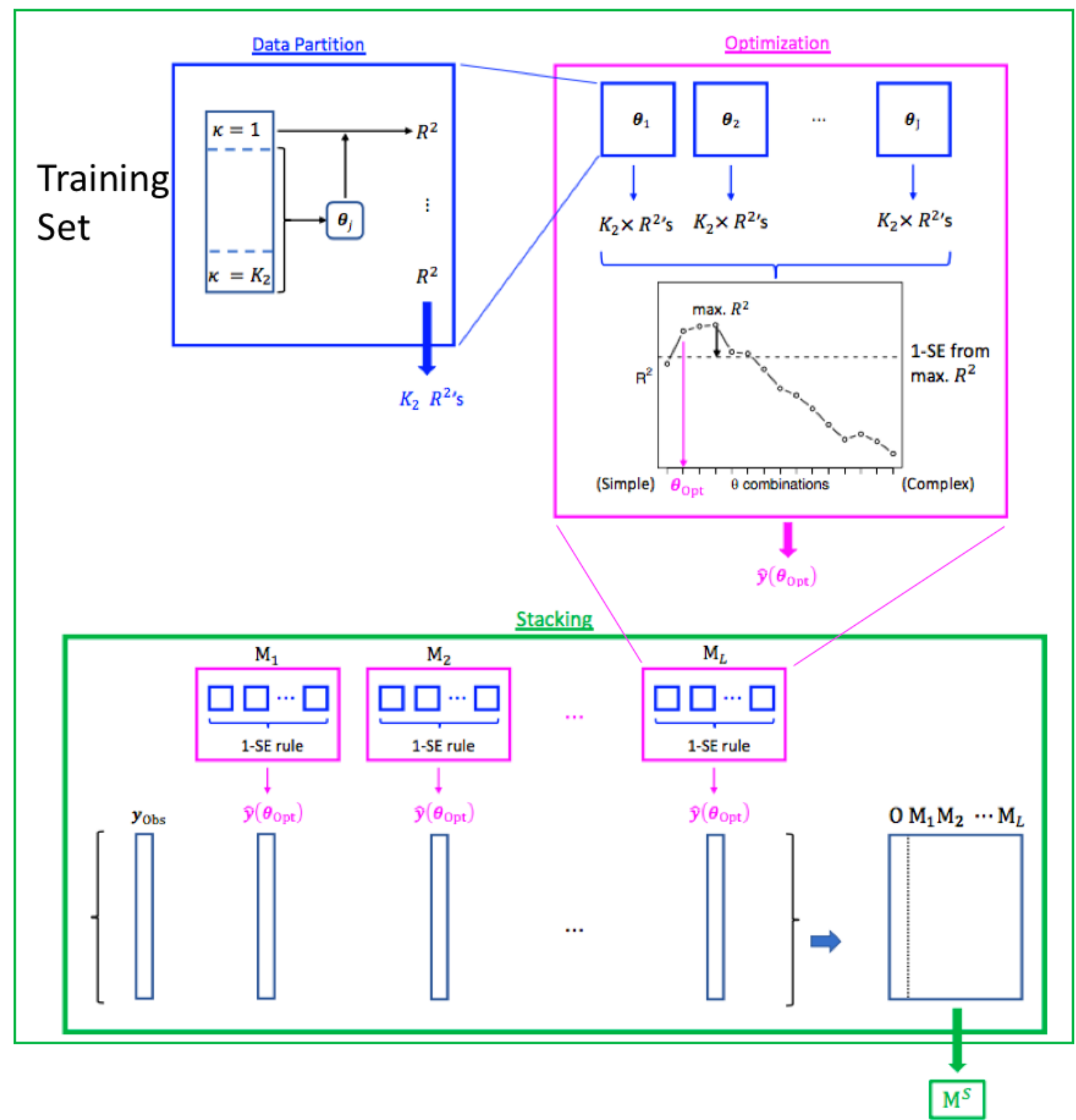

Figure S2. The inner-loop of repeated nested CV. In the inner loop, each training set from the outer loop was further partitioned into $K_{2}$ parts indexed by $\kappa$. A model with a tuning parameter combination $\boldsymbol{\theta}_{\mathrm{j}}$ was trained on $\left(K_{2}-1\right)$ parts, and then this model was applied to 
predict the held-out set. The predicted values were then compared to the observed values by R-square. Iterating across $K_{2}$ folds led to $K_{2}$ values of R-square. The process was repeated for all parameter combinations, and the optimal tuning parameter combination $\left(\boldsymbol{\theta}_{\text {Opt }}\right)$ was determined by the "one-SE" rule. This optimal parameter combination was applied to the whole training set and gave the predicted values as $\widehat{\boldsymbol{y}}\left(\boldsymbol{\theta}_{\mathrm{Opt}}\right)$. This process was then repeated for all machine learning methods. The predicted values across machine learning methods were then combined with observed values to build a stack model $\mathrm{M}^{\mathrm{S}}$.

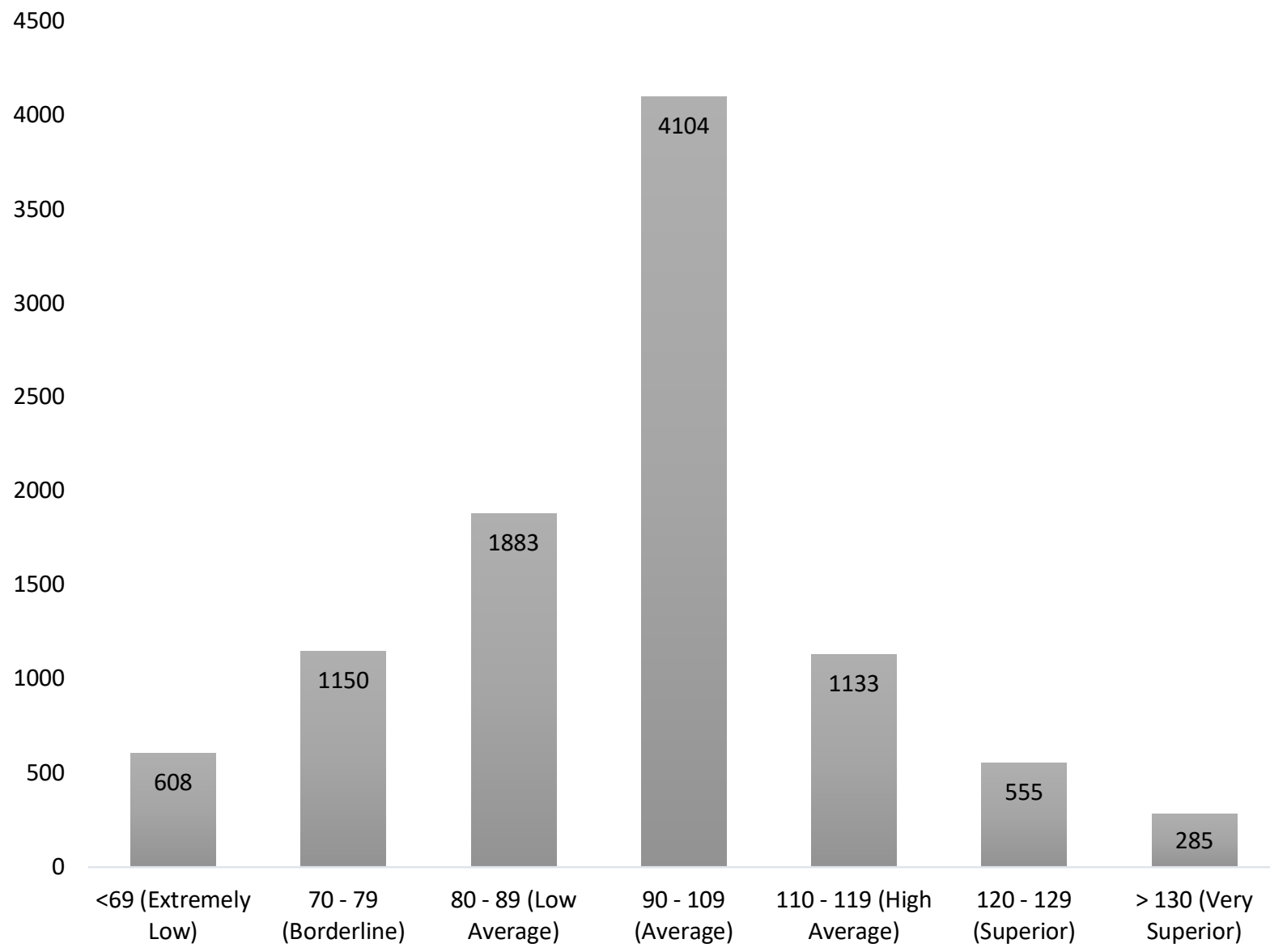

Figure S3. Distribution of age-corrected Fluid Cognitive Functioning (FCF) composite scores. 


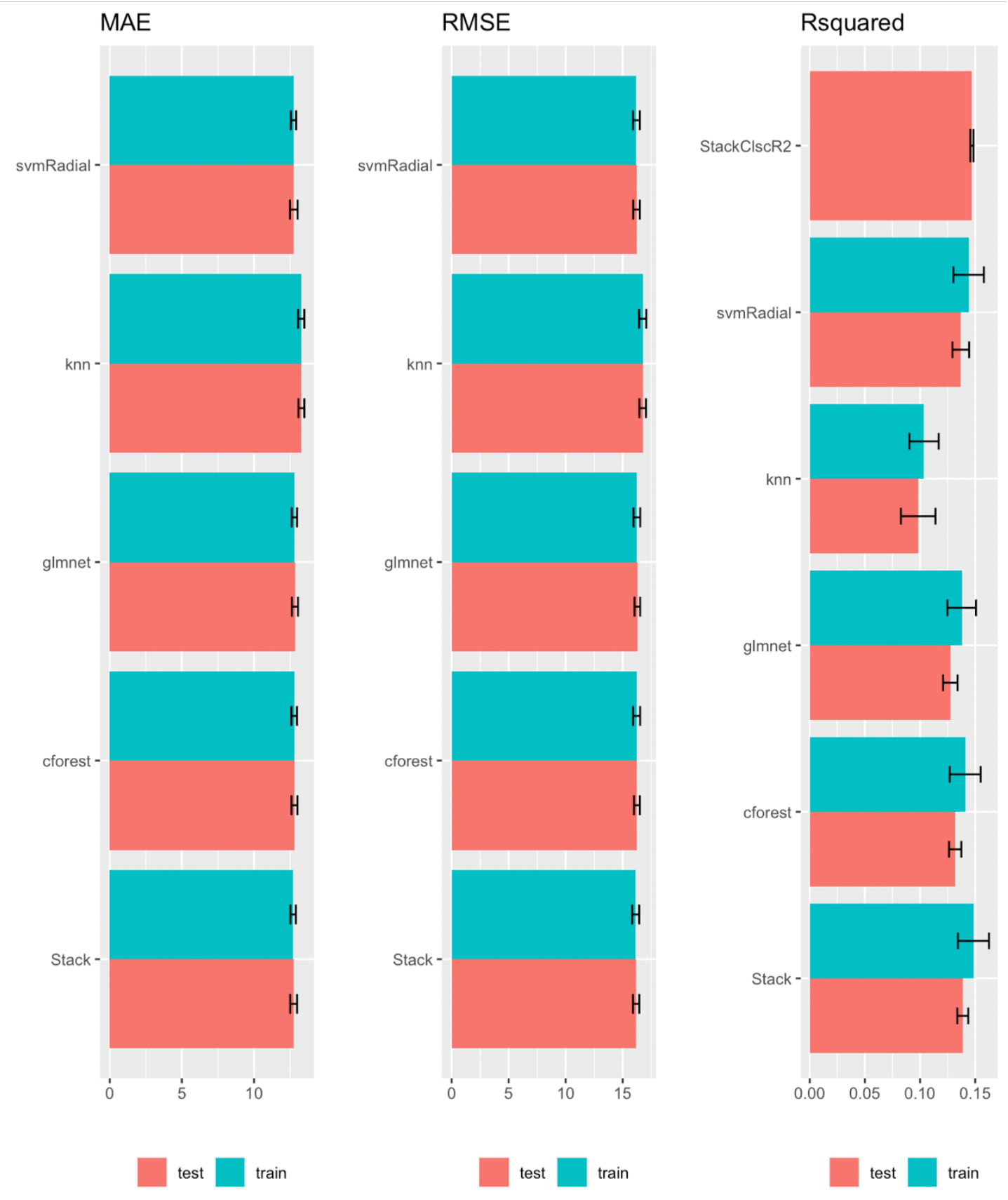

Figure S4. Percent of variance explained for age corrected fluid cognitive functioning scores by 47 demographic, psychological, and environmental predictors $(n=9,719)$. glmnet: Elastic net; cforest: conditional random forest; knn: k-nearest neighbors; svmRadial: support vector machine; StackClscR2: combined (stacked) method. Error bars represent 95\% confidence intervals. 


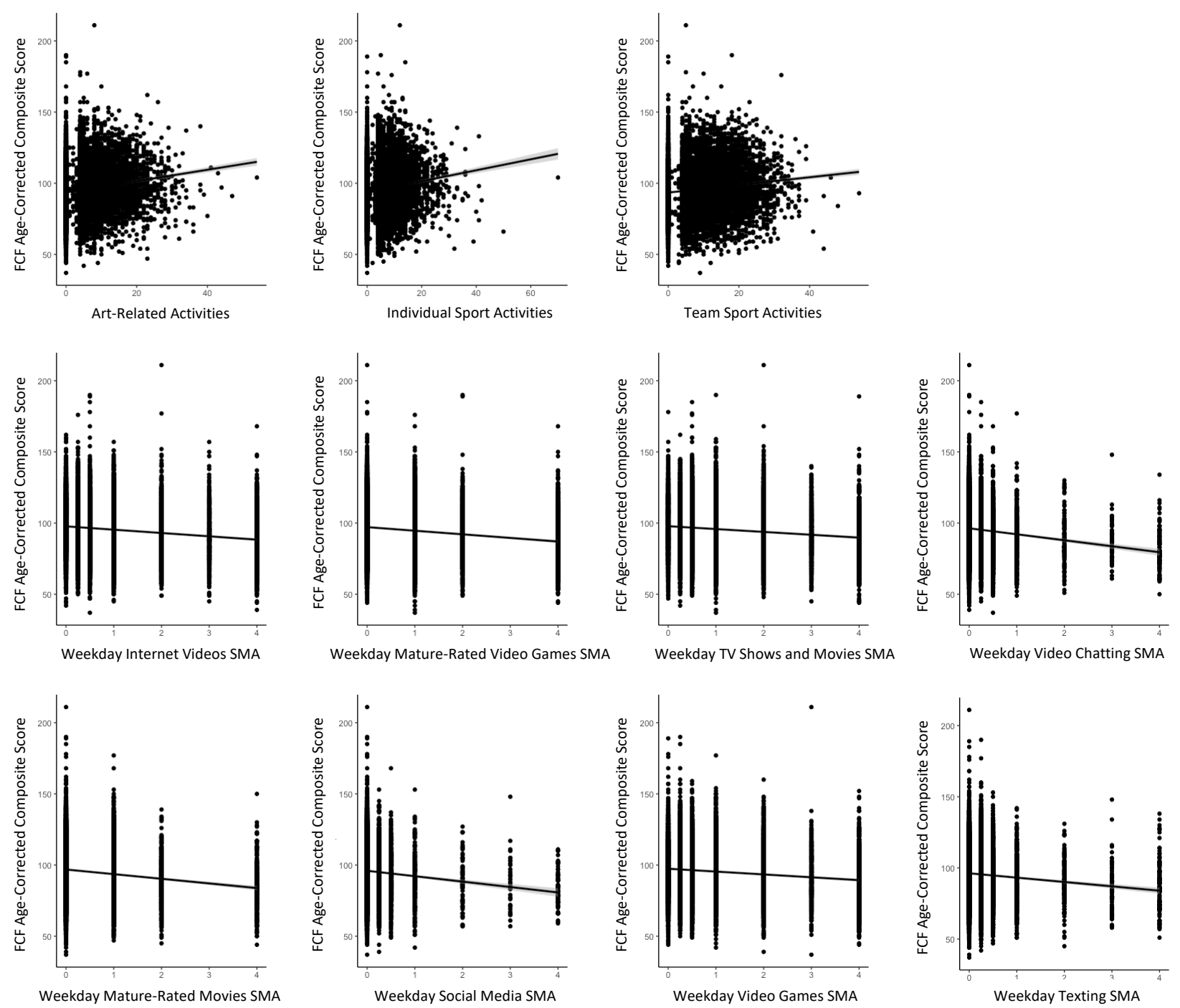

Figure S5. Scatter plots showing the relationship between potentially modifiable variables and Fluid Cognitive Fucntioning (FCF) composite scores. Abbreviations: SMA, screen media activity. 


\section{References}

Achenbach, T. M. (1997). Manual for the young adult self-report and young adult behavior checklist: University of Vermont, Department of Psychiatry.

Achenbach, T. M. (2009). The Achenbach System of Emprically Based Assessment (ASEBA): Development, Findings, Theory and Applications. In. University of Vermong Research Center for Children, Youth, and Families, Burlington, VT.

Achenbach, T. M., \& Ruffle, T. M. (2000). The Child Behavior Checklist and related forms for assessing behavioral/emotional problems and competencies. Pediatrics in review, 21(8), 265-271.

Arthur, M. W., Briney, J. S., Hawkins, J. D., Abbott, R. D., Brooke-Weiss, B. L., \& Catalano, R. F. (2007). Measuring risk and protection in communities using the communities that care youth survey. Eval. Program Plann., 30, 197-211.

Bagot, K., Matthews, S. A., Mason, M., Squeglia, L. M., Fowler, J., Gray, K., . . Godino, J. (2018). Current, future and potential use of mobile and wearable technologies and social media data in the $A B C D$ study to increase understanding of contributors to child health. Developmental cognitive neuroscience, 32, 121-129.

Bagot, K. S., Matthews, S. A., Mason, M., Squeglia, L. M., Fowler, J., Gray, K., . . Patrick, K. (2018). Current, future and potential use of mobile and wearable technologies and social media data in the $A B C D$ study to increase understanding of contributors to child health. Dev Cogn Neurosci, 32, 121-129. Retrieved from https://www.ncbi.nlm.nih.gov/pubmed/29636283. doi:10.1016/j.den.2018.03.008

Barch, D. M., Albaugh, M. D., Avenevoli, S., Chang, L., Clark, D. B., Glantz, M. D., . . YurgelunTodd, D. (2018). Demographic, physical and mental health assessments in the adolescent brain and cognitive development study: rationale and description. Dev Cogn Neurosci, 32, 55-66.

Barch, D. M., Albaugh, M. D., Avenevoli, S., Chang, L., Clark, D. B., Glantz, M. D., ... Sher, K. J. (2018). Demographic, physical and mental health assessments in the adolescent brain and cognitive development study: Rationale and description. Dev Cogn Neurosci, 32, 5566. Retrieved from https://www.ncbi.nlm.nih.gov/pubmed/29113758. doi:10.1016/j.dcn.2017.10.010

Barsaglini, A., Sartori, G., Benetti, S., Pettersson-Yeo, W., \& Mechelli, A. (2014). The effects of psychotherapy on brain function: a systematic and critical review. Progress in Neurobiology., 114, 1-14.

Bergstra, J., \& Bengio, Y. (2012). Random search for hyper-parameter optimization. Journal of Machine Learning Research, 13(Feb), 281-305.

Bruni, O., Ottaviano, S., Guidetti, V., Romoli, M., Innocenzi, M., Cortesi, F., \& Giannotti, F. (1996). The Sleep Disturbance Scale for Children (SDSC) Construct ion and validation of an instrument to evaluate sleep disturbances in childhood and adolescence. Journal of sleep research, 5(4), 251-261.

Bruni, O., Ottaviano, S., Guidetti, V., Romoli, M., Innocenzi, M., Cortesi, F., \& Giannotti, F. (1996). The Sleep Disturbance Scale for Children (SDSC). Construction and validation of an instrument to evaluate sleep disturbances in childhood and adolescence. J. Sleep Res, 5(4), 251-261. 
Caputo, B., Sim, K., Furesjo, F., \& Smola, A. (2002). Appearance-based object recognition using SVMs: which kernel should I use? Paper presented at the Proc of NIPS workshop on Statistical methods for computational experiments in visual processing and computer vision, Whistler.

Carver, C. S., \& White, T. L. (1994). Behavioral inhibition, behavioral activation, and affective responses to impending reward and punishment: the BIS/BAS scales. Journal of Personality and Social Psychology, 67(2), 319.

CDC. (2016a). Youth Risk Behavior Survey. Retrieved from https://www.cdc.gov/healthyyouth/data/yrbs/.

CDC. (2016b). Youth Risk Behavior Survey. Retrieved from https://www.cdc.gov/healthyyouth/data/yrbs/

CDC (Division of Nutrition, P. A. a. O. (2016). Anthropometry Procedures Manual. Retrieved from https://wwwn.cdc.gov/nchs/data/nhanes/2015-2016/manuals/2016 Anthropometry_Procedures_Manual.pdf.

Chilcoat, H. D., \& Anthony, J. C. (1996). Impact of parent monitoring on initiation of drug use through late childhood. J. Am. Acad. Child Adolesc. Psychiatry, 35, 91-100.

Chilcoat, H. D., \& Anthony, J. C. (1996). Impact of parent monitoring on initiation of drug use through late childhood. Journal of the American Academy of Child \& Adolescent Psychiatry, 35(1), 91-100.

Diemer, M. A., Mistry, R. S., Wadsworth, M. E., Lopez, I., \& Reimers, F. (2012). Best practices in conceptualizing and measure social class in psycholgocial research. Anal. Soc. Issues Public Policy, 13(1), 77-113.

Filzmoser, P., Liebmann, B., \& Varmuza, K. (2009). Repeated double cross validation. Journal of Chemometrics: A Journal of the Chemometrics Society, 23(4), 160-171.

Hothorn, T., Hornik, K., \& Zeileis, A. (2006). Unbiased recursive partitioning: A conditional inference framework. Journal of Computational and Graphical statistics, 15(3), 651-674.

Huppertz, C., Bartels, M., de Zeeuw, E. L., van Beijsterveldt, C. E. M., Hudziak, J. J., Willemsen, G., ... de Geus, E. J. C. (2016). Individual differences in exercise behavior: stability and change in genetic and environmental determinants from age 7-18. Behav. Genet., 46(5), 665-679.

Keller, J. M., Gray, M. R., \& Givens, J. A. (1985). A fuzzy k-nearest neighbor algorithm. IEEE transactions on systems, man, and cybernetics(4), 580-585.

Kobak, K. A., \& Kaufman, J. (2015). Ksads-comp. Madison, WI: Center for Telepsychology.

Kuhn, M. (2014). Futility analysis in the cross-validation of machine learning models. arXiv preprint arXiv:1405.6974.

Lisdahl, K. M., Sher, K. J., Conway, K. P., Gonzalez, R., Ewing, S. W. F., Nixon, S. J., . . . Heitzeg, M. (2018). Adolescent brain cognitive development (ABCD) study: overview of substance use assessment methods. Developmental cognitive neuroscience, 32, 80-96.

Lisdahl, K. M., Sher, K. J., Conway, K. P., Gonzalez, R., Feldstein Ewing, S. W., Nixon, S. J., .. . Heitzeg, M. (2018). Adolescent brain cognitive development (ABCD) study: Overview of substance use assessment methods. Dev Cogn Neurosci, 32, 80-96. Retrieved from https://www.ncbi.nlm.nih.gov/pubmed/29559216. doi:10.1016/j.dcn.2018.02.007

Lynam, D. R. (2013). Development of the Short Form of the UPPS-P Impulsive Behavior Scale. Retrieved from Unpublished Technical Report: 
Moos, R. H., \& Moos, B. S. (1994). Family environment scale manual: Consulting Psychologists Press.

Pagliaccio, D., Luking, K. R., Anokhin, A. P., Gotlib, I. H., Hayden, E. P., Olino, T. M., ... Barch, D. M. (2016). Revising the BIS/BAS Scale to study development: measurement invariance and normative effects of age and sex from childhood through adulthood. Psychol. Assess, 28(4), 439-442.

Puig-Antich, J., \& Ryan, N. (1986). Kiddie schedule for affective disorders and schizophrenia. Pittsburgh, PA: Western Psychiatric Institute.

Rice, J. P., Reich, T., Bucholz, K. K., Neuman, R. J., Fishman, R., Rochberg, N., . . Begleiter, H. (1995). Comparison of direct interview and family history diagnoses of alcohol dependence. Alcoholism: Clinical and Experimental Research, 19(4), 1018-1023.

Rice, J. P., Reich, T., Bucholz, K. K., Neuman, R. J., Fishman, R., Rochberg, N., . . . Begleiter, H. (1995). Comparison of direct interview and family history diagnoses of alcohol dependence. Alcohol. Clin. Exp. Res, 19(4), 1018-1023.

Schaefer, E. S. (1965). A configurational analysis of children's reports of parent behavior. J. Consult. Psychol., 29, 552-557.

Singh, G. K. (2003). Area deprivation and widening inequalities in US mortality, 1969-1998. Am J Public Health, 93, 1137-1143.

Stone, M. (1974). Cross-validatory choice and assessment of statistical predictions. Journal of the Royal Statistical Society: Series B (Methodological), 36(2), 111-133.

Stover, P. J., Harlan, W. R., Hammond, J. A., Hendershot, T., \& Hamilton, C. M. (2010). PhenX: a toolkit for interdisciplinary genetics research. Current opinion in lipidology, 21(2), 136140.

Stover, P. J., Harlan, W. R., Hammond, J. A., Hendershot, T., \& Hamilton, C. M. (2010). PhenX: a toolkit for interdisciplinary genetics research. Curr Opin Lipidol, 21(2), 136-140. Retrieved from https://www.ncbi.nlm.nih.gov/pubmed/20154612. doi:10.1097/MOL.0b013e3283377395

Suykens, J. A., \& Vandewalle, J. (1999). Least squares support vector machine classifiers. Neural processing letters, 9(3), 293-300.

Toolkit, P. (2016). Neighborhood Safety (\#210900 \& 210901). Retrieved from https://www.phenxtoolkit.org/toolkit content/PDF/PX210901.pdf.

Varma, S., \& Simon, R. (2006). Bias in error estimation when using cross-validation for model selection. BMC bioinformatics, 7(1), 91.

Whiteside, S. P., Lynam, D. R., Miller, J. D., \& Reynolds, S. K. (2005). Validation of the UPPS impulsive behaviour scale: a four-factor model of impulsivity. European Journal of Personality: Published for the European Association of Personality Psychology, 19(7), 559-574.

Youngstrom, E. A., Frazier, T. W., Demeter, C., Calabrese, J. R., \& Findling, R. L. (2008). Developing a 10-item mania scale from the Parent General Behavior Inventory for children and adolescents. J. Clin. Psychiatry, 69(5), 831-839.

Youngstrom, E. A., Frazier, T. W., Demeter, C., Calabrese, J. R., \& Findling, R. L. (2008). Developing a ten item mania scale from the parent general behavior inventory for children and adolescents. The Journal of clinical psychiatry, 69(5), 831. 
Zapolski, T. C., Stairs, A. M., Settles, R. F., Combs, J. L., \& Smith, G. T. (2010). The measurement of dispositions to rash action in children. Assessment, 17(1), 116-125.

Zucker, R. A., Gonzalez, R., Ewing, S. W. F., Paulus, M. P., Arroyo, J., Fuligni, A., . . Wills, T. (2018). Assessment of culture and environment in the Adolescent Brain and Cognitive Development Study: Rationale, description of measures, and early data. Developmental cognitive neuroscience, 32, 107-120.

Zucker, R. A., Gonzalez, R., Feldstein Ewing, S. W., Paulus, M. P., Arroyo, J., Fuligni, A., . . . Wills, T. (2018). Assessment of culture and environment in the Adolescent Brain and Cognitive Development Study: Rationale, description of measures, and early data. Dev Cogn Neurosci, 32, 107-120. Retrieved from https://www.ncbi.nlm.nih.gov/pubmed/29627333. doi:10.1016/j.dcn.2018.03.004 